\title{
ICRF antenna matching systems with ferrite tuners for the
}

\section{Alcator C-Mod tokamak}

\author{
Y. Lin, A. Binus, S. J. Wukitch, P. Koert, R. Murray, and A. Pfeiffer \\ MIT Plasma Science and Fusion Center, Cambridge, MA 02139, USA \\ Email: ylin@psfc.mit.edu
}

\begin{abstract}
Real-time fast ferrite tuning (FFT) has been successfully implemented on the ICRF antennas on Alcator C-Mod. The former prototypical FFT system on the E-port 2-strap antenna has been upgraded using new ferrite tuners. A new FFT system with two ferrite tuners and one fixedlength stub has been installed on the transmission line of the D-port 2-strap antenna. These two systems are able to achieve and maintain the reflected power to the transmitters to less than $1 \%$ in real time under almost all plasma conditions and help ensure reliable high power operation of the antennas. The loading insensitivity feature vs. plasma conditions of the innovative fieldaligned (FA) 4-strap antenna on the J-port allows us to significantly improve the matching by installing a carefully designed stub on each of the two transmission lines. The reduction of the RF voltages in the transmission lines has enabled the J-port FA antenna to deliver 3.7 MW RF power to plasmas out of $4 \mathrm{MW}$ source power. The matching on the J-port antenna can be further improved by adding a single ferrite tuner under real-time control on each transmission line and this scheme will be implemented in the near future.
\end{abstract}

Keywords: ICRF, antenna matching, tokamak, Alcator C-Mod, ferrite tuner, real-time control 


\section{Introduction}

Ion cyclotron range of frequencies (ICRF) heating is an important auxiliary heating method in tokamaks, and it is part of the heating systems on ITER to heat the fusion ions, boost fusion reaction and sustain fusion burn. ICRF has some clear advantages over other heating systems: the ICRF fast waves can easily penetrate the plasma and can be strongly absorbed; and inexpensive, reliable power sources are readily available.

The power transmitter for ICRF heating is typically a MW-level system with tetrode vacuum tubes as the final power amplifier operating at the frequency range of tens of $\mathrm{MHz}$. The output impedance of the tubes and the characteristic impedance of the transmission line are typically 50

$\Omega$. The radio frequency (RF) power from the transmitter is delivered via a transmission line to the ICRF antenna inside the tokamak. The antenna is essentially radiating current straps, physically quite close to the plasma, and have rather small loading, $\sim 0.1-10 \Omega$. The antenna loading also varies (sometimes rapidly) in response to the changes in plasmas. Therefore, a matching network is required between the transmitter and the antenna so that ideally the transmitter would output RF power to an equivalently matched $50 \Omega$ load. Maintaining the antenna matching under varying (especially fast and transient) plasma loading remains an important but not yet fully resolved technical issue for the ICRF community.

Many types of matching networks have been developed on different tokamaks. The conventional approach is to use stubs and phase-shifters (line stretchers) that have mechanically variable lengths. These systems are usually slow and they are not designed to vary during plasma discharge during high RF power operation. As a result, if plasma loading changes rapidly or varies in a large range, the transmitter would be mismatched. Many groups have studied and 
implemented matching systems that can be used in real-time, including frequency modulation on JET [1], dielectric liquid stubs on EAST [2] and LHD [3], ferrite tuners on Alcator C-Mod [4], EAST [5] and KSTAR [6]. In this paper, we report the recent progress on the implementation and performance of real time fast matching system using ferrite tuners on the Alcator C-Mod tokamak.

Alcator C-Mod [7] is a compact high field tokamak: $\mathrm{B}_{\mathrm{t}} \leq 8.1 \mathrm{~T}, \mathrm{R} \approx 0.68 \mathrm{~m}, \mathrm{a} \approx 0.22 \mathrm{~m}, \mathrm{I}_{\mathrm{p}} \approx 0.4$ $-1.3 \mathrm{MA}, \mathrm{n}_{\mathrm{e} 0} \approx 0.5-4 \times 10^{20} \mathrm{~m}^{-3}$, and $\mathrm{T}_{\mathrm{e} 0} \leq 9 \mathrm{keV}$. For a typical plasma with $\mathrm{B}_{\mathrm{t}} \sim 5.4 \mathrm{~T}$, the ICRF system at $\sim 80 \mathrm{MHz}$ heats plasma using the minority heating scenario, i.e., the RF frequency equals to the fundamental cyclotron resonance frequency of the minority $\mathrm{H}$ ions. There are three ICRF antennas on the tokamak, labeled as D-port antenna, E-port antenna and Jport antenna respectively, corresponding to the horizontal ports where the antennas are located. The D-port and E-port antennas [8,9] are two-strap antennas, each of which is fed by one $2 \mathrm{MW}$ transmitter and operated at 80.5 and $80 \mathrm{MHz}$ respectively. The J-port antenna is an innovative field aligned (FA) 4-strap antenna, fed by two $2 \mathrm{MW}$ transmitters and operated from $50-80$ MHz. Further description of the FA J-port antenna can be found here.[10] Prior to year 2008, the matching system on each transmission line consisted of a mechanical stub tuner and a mechanical phase shifter. In Ref. [4], we reported the successful implementation of a prototypical fast ferrite tuning (FFT) system on the E-port antenna, commissioned in 2009. The real-time matching system included two ferrite tuners and a real-time control computer that was incorporated into the plasma control system.[11] The ferrite tuners used in the prototypical system were not designed for the operational parameters for Alcator C-Mod; instead, they had been designed for a lower frequency, and also the range of variable electrical length was quite limited at $80 \mathrm{MHz}$.[12] In this paper, we report the successful installation of new matching 
systems on both D-port and E-port antennas using new ferrite tuners that were designed and manufactured to meet the specifications for the ICRF operation on Alcator C-Mod. We also report the modification on the transmission lines of the FA J-port antenna to improve reliability and the design of a simpler ferrite tuning system for the FA J-port antenna.

The paper is organized as follows: Section 2 describes the new FFT systems on the D-port and E-port antennas; Section 3 shows the performance of the FFT systems; Section 4 describes the performance of the pre-match stubs on the FA J-port antenna and also the design of a real time ferrite tuning system on the FA J-port antenna, followed by Summary in Section 5.

\section{Design of the Fast ferrite tuning system on $D$ and $E$ antennas}

An issue at stake for ICRF operation in a tokamak is that the antenna loading varies in time and often the variation in loading can be large and fast. Although the transmitter may be tolerant of a slight mismatch, generally it is not allowed to operate when the mismatch is larger than the threshold that is pre-set for arc detection. Shutting-off the RF power source when a significant mismatch is detected has been mandatary in all the ICRF heating systems.[13] Note that although not all mismatches are caused by real arcs, arcs at high voltage points in the system almost always generate mismatch. As a result, large mismatches caused by loading variation not only limit the transmitter performance at high power, but they can also severely reduce the duty factor of ICRF heating in plasmas. The loss of experimental time can be costly for present-day tokamaks, and in fusion reactors, mismatches should ideally be avoided all the time. Therefore, an ideal ICRF matching system should transform the antenna loading into a steady and stable near-perfect match as seen by the transmitter and should be able to handle the changing antenna

loading in real time under nearly all plasma conditions. The matching system should also be able 
to handle the high RF voltage during high power (and long pulse) operation while at the same time introducing additional RF power losses as small as possible.

Transmission line matching using two or more stubs is a standard RF engineering application. The system we have implemented on Alcator C-Mod is a three-stub system. The schematic layout is shown in Figure 1 and the picture is shown in Figure 2. The stub closest to the antenna, Stub-3, has a fixed length, and the other two, Stub-1 and Stub-2, are variable stubs, each of which is a combination of a fixed-length transmission line section and a ferrite tuner. Because the structures and loading of the D-port and E-port antennas are rather similar, we use the same added lengths to the tuners. The total length of Stub-1, $\mathrm{L}_{1}=1.29 \mathrm{~m}+\mathrm{L}_{\text {tuner }}$, and Stub-2, $\mathrm{L}_{2}=$ $0.71 \mathrm{~m}+\mathrm{L}_{\text {tuner2}}$, where $\mathrm{L}_{\text {tuner1 }}$ and $\mathrm{L}_{\text {tuner2 }}$ are the equivalent lengths of the tuners (discussed below). The length between the two variable stubs is set to be $3 \lambda / 8, \mathrm{~L}_{12} \approx 1.40 \mathrm{~m}$ for both antennas. The distance $3 \lambda / 8$ is a standard choice for double-stub matching, where $\mathrm{L}_{12}$ can be $3 \lambda / 8+n \lambda / 2$ or $\lambda / 8+n \lambda / 2$, and $n$ is an arbitrary integer. Several iterations have been taken for both systems in order to optimize the locations and lengths of all the stubs. There are differences in the locations and lengths of Stub-3 due to space and other consideration in making transmission line connections (Figure 2): $\mathrm{L}_{23}=3.88 \mathrm{~m}$ and $\mathrm{L}_{3}=1.57 \mathrm{~m}$ for the D-port antenna and $\mathrm{L}_{23}=2.31 \mathrm{~m}$ and $\mathrm{L}_{3}=0.51 \mathrm{~m}$ for the $\mathrm{E}$ antenna. Because many elbows and $\mathrm{T}$-connectors are used in the setup (see Figure 2), the lengths quoted here are equivalent electrical lengths calculated from the phase data from directional couplers measurement, which are slightly different than the geometrical lengths. 


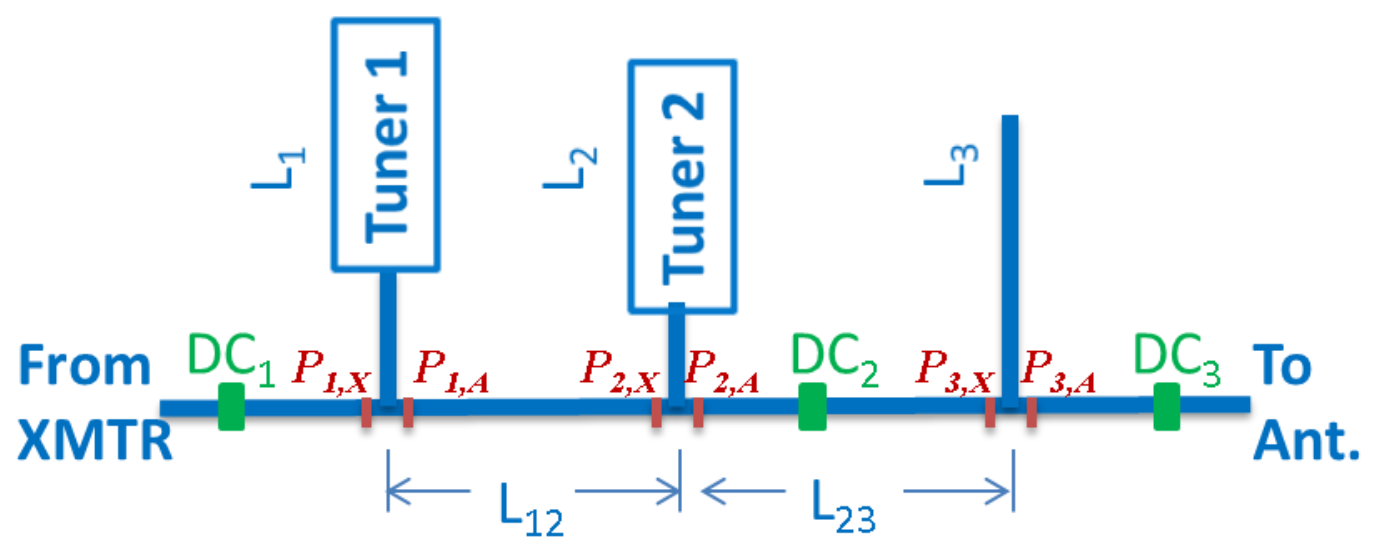

Figure 1 Layout of the FFT system. DCs are directional couplers measuring the RF power and phase. The lengths are described in the text. $\mathbf{P}_{*, *}$ are the points on the transmitter side and antenna side of the stubs. "XMTR" stands for "transmitter" and "ant." stands for "antenna".

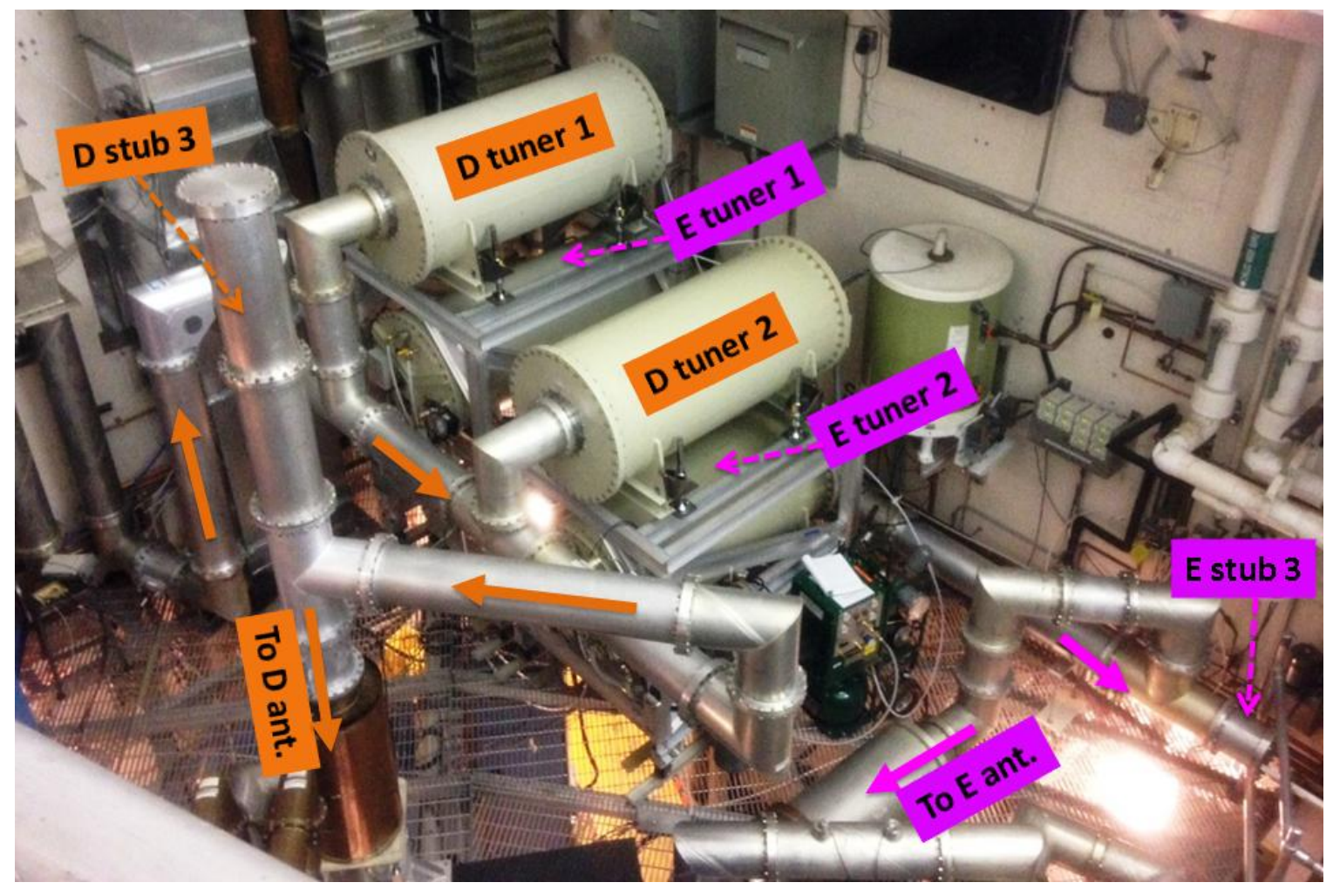

Figure 2 Picture of the FFT system. Tuners and stubs are labeled. Also the RF power flow is indicated.

Here we discuss the characteristics of the ferrite tuners. The propagation speed of RF waves in a transmission line is determined by the electric permittivity $\varepsilon$ and the magnetic permeability $\mu$. 
The principle of the ferrite tuners is that the $\mu$ of the ferrite tiles inside the tuners can be varied in real time, and as a result, the tuners are capable of following the loading variation of ICRF antennas due to changes in the plasma. In this paper, we use the equivalent electrical length to describe the length of a ferrite tuner: The equivalent electrical length for the RF wave to propagate in one oscillation time period has the equivalent electrical length of one wavelength in vacuum. The ferrite tiles are immersed in a permanent magnetic field. To change the equivalent length of a line section filled with ferrite tiles, we vary $\mu$ by adding a bias magnetic field onto the permanent ambient field, which moves ferrite tiles to different positions on the magnetization curve. Varying this bias field is achieved by varying current in current coils surrounding the tiles. Thus the ferrite tuners behave like variable length stubs that can be simply controlled by the coil currents from the outside. The new ferrite tuners, made by Advanced Ferrite Technology GmbH in Germany, have larger range of variable electrical lengths at $80 \mathrm{MHz}$ than the prototypical ones reported earlier. They are specifically made for the conditions on Alcator C-Mod: optimum operation frequency at $80 \mathrm{MHz}$, and their electrical length can vary as much as $0.49 \mathrm{~m}$ for the coil current ranging from $-150 \mathrm{~A}$ to $+150 \mathrm{~A}$. The equivalent electrical length of the tuners is $\sim 1.91 \mathrm{~m}$ to $\sim 1.42 \mathrm{~m}$ in this range of coil current, where the initial equivalent length at zero coil current is $\sim 1.63 \mathrm{~m}$. The response curve of the electrical length vs. coil current of each tuner is measured during bench tests (i.e., very low RF power) and then the curve is spline fit to a $3^{\text {rd }}$ degree polynomial by the control algorithm during real time computation. Before operating with high RF power, the tuners are dried by flowing dry nitrogen gas, and then filled with 2 atm (1 atm relative pressure) sulfur hexafluoride $\left(\mathrm{SF}_{6}\right)$ after the moisture level has become very low. The tuners are designed to be able to handle $>40 \mathrm{kV}$ RF voltage. Continuous water cooling on the ferrite tiles ensures the tuners to be able to handle high power and long pulse. The flow rate 
and temperature of the cooling water and the signal from an optical arc detector are built into the operation safety and protection via a programmable logic controller (PLC). The power supplies for the coil currents are controlled in real-time during plasmas by a Linux-server-based controller.

The operational details of the system can be illustrated on a Smith chart [14], which is a standard tool for transmission line representation. In Figure 3, we show a rotated Smith admittance chart $\mathrm{Y} \equiv 1 / \mathrm{Z}=\mathrm{G}+j \mathrm{~B}$, where $\mathrm{Z}$ is impedance, $\mathrm{G}$ is conductance and $\mathrm{B}$ is susceptance, normalized to $\mathrm{G}_{0}=1 / \mathrm{Z}_{0}$ and $\mathrm{Z}_{0}=50 \Omega$ and $j$ is the imaginary unit. The $\mathrm{X}$-axis and the $\mathrm{Y}$-axis show the negative voltage reflection coefficient $-\operatorname{Re}(\Gamma)$ and $-\operatorname{Im}(\Gamma)$ respectively. Note that at a given location on a transmission line, admittance $Y=(1-\Gamma) /(1+\Gamma)$, and the power reflection coefficient is $|\Gamma|^{2}$. The admittance of a lossless stub (with a short) $\mathrm{Y}_{\text {stub }}$ has $\mathrm{G}=0$ and $\mathrm{B}=$ $-1 / \tan (\beta \mathrm{L})$, where $\beta=2 \pi / \lambda$ and $\lambda$ is the wavelength in vacuum and $\mathrm{L}$ is the equivalent electrical length of the stub. In our calculation for impedance matching, we use the approximation that the ferrite tuners are lossless while in reality the ferrite tiles are lossy under some conditions. The admittance on the transmitter side of the stub, $\mathrm{Y}_{\mathrm{X}}$ and the admittance on the antenna side of the stub, $\mathrm{Y}_{\mathrm{A}}$, are related by a simple equation, $\mathrm{Y}_{\mathrm{X}}=\mathrm{Y}_{\mathrm{A}}+\mathrm{Y}_{\text {stub. }}$ As a result, because only susceptance B component of $\mathrm{Y}$ is changed, a stub would move a point from the antenna side to the transmitter side via a circle of constant conductance $\mathrm{G}$ (one of the circles that are tangential to the right $\mathrm{Y}$-axis on the Smith chart). On the other hand, a transmission line section with normalized $Z=1$ (or $G=1$ ), i.e., $Z=Z_{0}=50 \Omega$, would move a point on a constant $|\Gamma|$ circle with only the argument (phase angle) of the complex $\Gamma$ are changed. The voltage standing wave ratio (VSWR) of the line section is determined by VSWR $=(1+|\Gamma|) /(1-|\Gamma|)$, and only at the very center of the Smith chart we have VSWR = 1 for perfect match. The matching process is also demonstrated in Figure 3. The point $\mathrm{P}_{2}$, $\mathrm{A}$, on the antenna side of Stub-2, would be transformed to 
$\mathrm{P}_{2, \mathrm{X}}$ on the transmitter side by Stub-2, and then move along a constant $|\Gamma|$ circle to $\mathrm{P}_{1, \mathrm{~A}}$ on the antenna side of Stub-1. $P_{1, A}$ is now on the $G=1$ circle. Stub- 1 then transforms point $P_{1, A}$, along $G$ $=1$ circle, to $\mathrm{P}_{1, \mathrm{x}}$ on the transmitter side of Stub-1, which is at the very center of the Smith chart for a perfect match. After this transformation, the transmitter would see a matched $50 \Omega$ load. The lengths $L_{1}$ and $L_{2}$ of Stub-1 and Stub-2 that are required to have perfection match can be calculated from a set of standard algebraic equations. All the points residing in the solidly filled area around $\mathrm{P}_{2, \mathrm{~A}}$ on the Smith chart can be transformed to the perfect match with the combination of the two ferrite tuners. All the points residing in the surrounding loosely filled regime can be successfully transformed to have $|\Gamma| \leq 0.1$ (i.e., VSWR $\leq 1.2$, and power reflection $\left.|\Gamma|^{2} \leq 1 \%\right)$. This level of match is well within the operational tolerance of the transmitter. The location and the length of the fixed stub, Stub-3, are carefully chosen so that under almost all plasma conditions, the reflection coefficient $\Gamma$ at point $\mathrm{P}_{2, \mathrm{~A}}$ would be inside the solidly filled area on the Smith chart. 


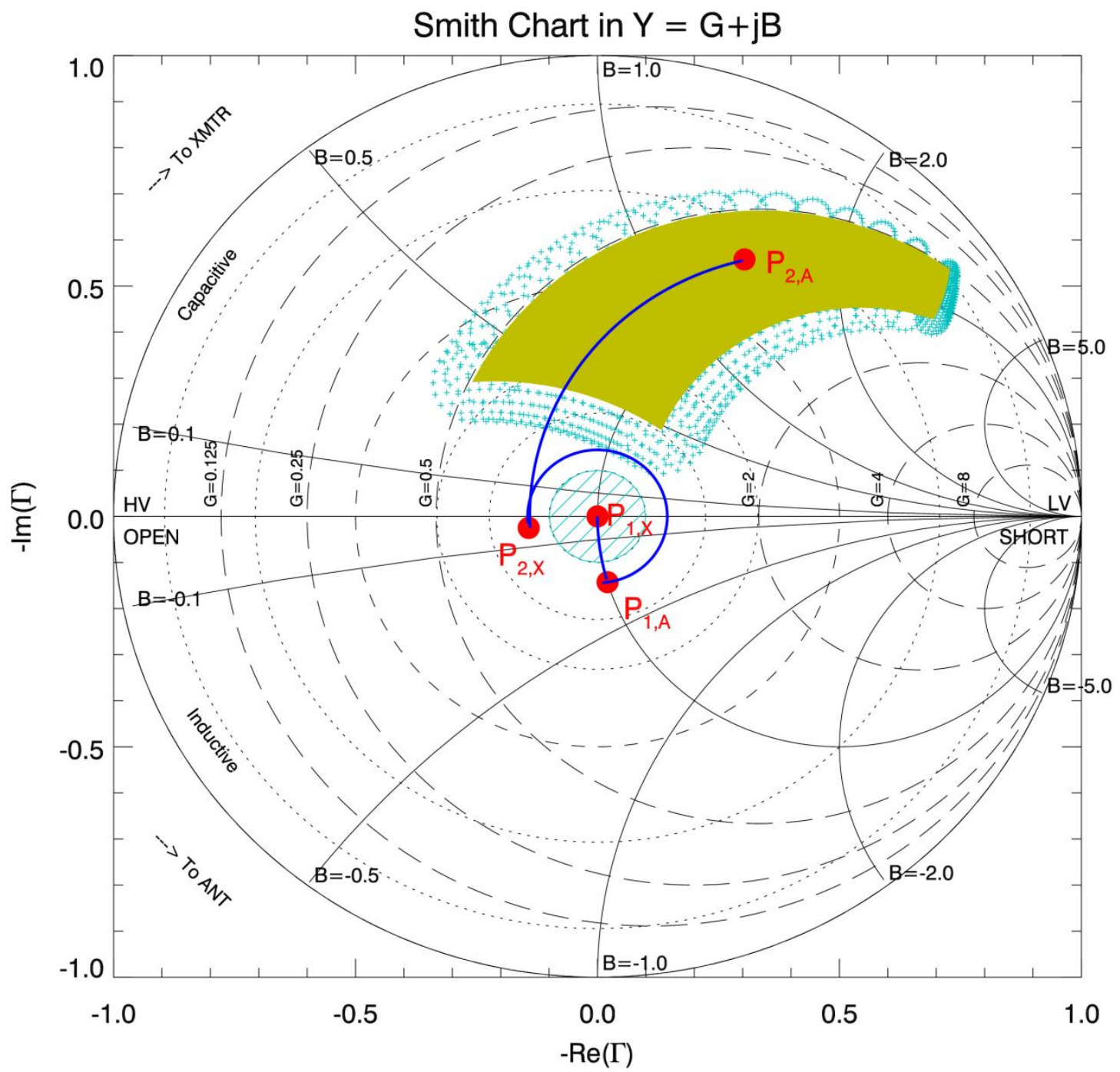

Figure 3 Design of the FFT system shown in a Smith admittance chart, where each point represents the negative generalized reflection coefficient $(-\Gamma)$ on a location of the transmission line. The points in red correspond to the locations on the transmission line labeled in Figure 1.

During a plasma discharge, the lengths $\mathrm{L}_{1}$ and $\mathrm{L}_{2}$ required to achieve the perfect match are calculated by a Linux-server based real time digital controller, which is integrated into the MDSplus data system for the Alcator C-Mod plasma operation.[15] The layout of the control system is shown in Figure 4. At the same time when the plasma control system loads the programmed 
parameters in the so-called INIT-phase of the plasma operation, the real-time ferrite tuner controller reads all the pre-set parameters from the MDS-plus data tree, for example, the response curves of tuner electrical length vs. coil current for each tuner, the location of the directional couplers related to the tuners, the calibration curves for the demodulators measuring RF power, etc. During the plasma pulse, the controller works in computation cycles. The time length of the cycle is also set in the MDS-plus data tree. At the beginning of each computation cycle, the controller reads, via the I/O ports, the real-time forward power, reflected power and phase from directional couplers along with the tuner coil currents, coil voltages and other status information for the ferrite tuners and their power supplies. With all the information in-hand, the algorithm first decides whether there is enough RF power in the transmission line. If there is enough power (usually the threshold is set at $30 \mathrm{~kW}$ ), the algorithm then starts the computation process by first calculating the existing stub electrical lengths. There are two sets of the results. One is based on the measurement of $\Gamma_{\mathrm{DC} 1}$ and $\Gamma_{\mathrm{DC} 2}$ (shown in Figure 1) and other geometrical parameters. Another set is calculated using the measured coil currents and the calibration curves of the tuner electrical lengths vs. coil currents. This first step helps check whether the tuners are malfunctioning or having a strong nonlinear response at high RF power. Both sets of calculations are stored in the data system. In most cases, the two sets agree with each other within a few centimeters. 


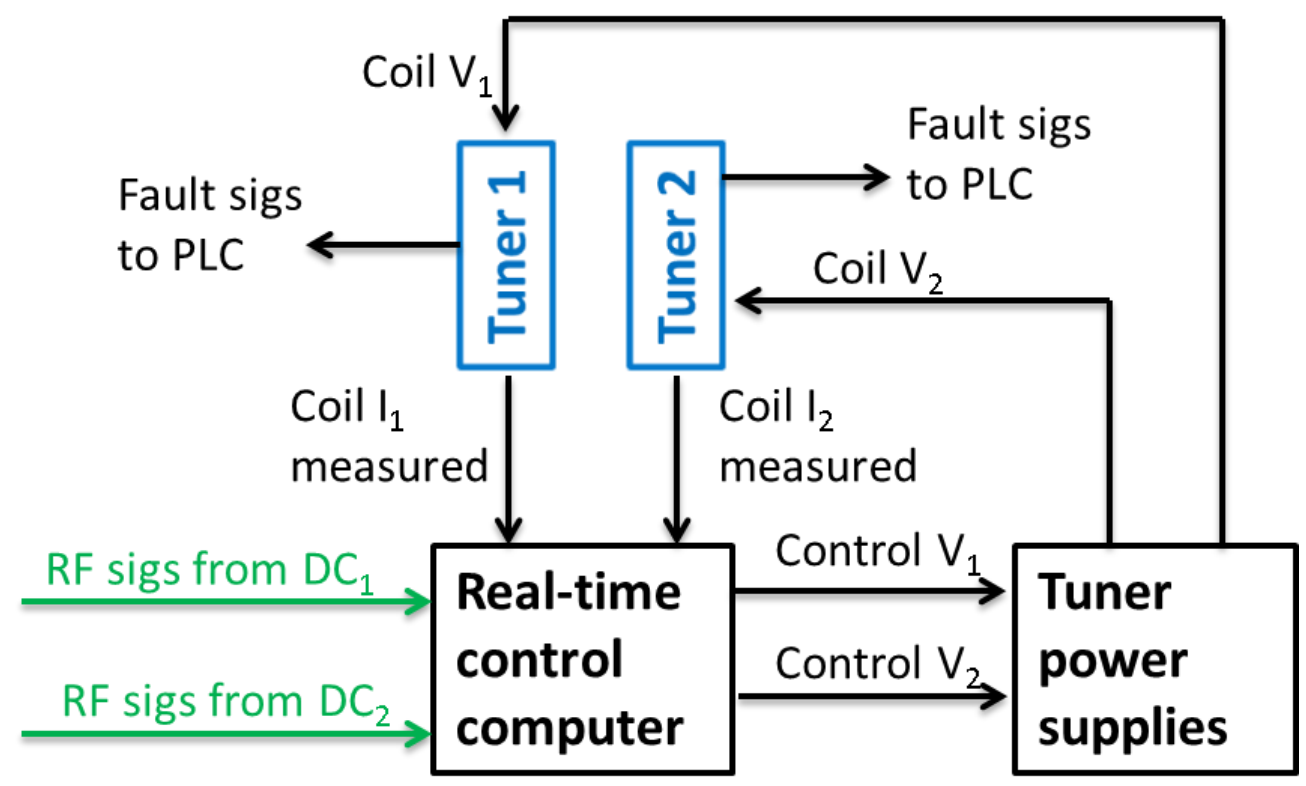

Figure 4 Layout of the control system.

The next step, the algorithm calculates the lengths $\mathrm{L}_{1}$ and $\mathrm{L}_{2}$ that are required to make $\Gamma_{\mathrm{DC} 1}=0$ assuming that $\Gamma_{\mathrm{DC} 2}$ would not change. The solutions can be derived and computed analytically given $\Gamma_{\mathrm{DC} 2}$ and the geometrical parameters of the system. By comparing the solutions with the existing stub lengths, the algorithm then decides how much changes in the coil currents are required and generates required control voltages for power supplies. At the end of the computation process, the control voltages are sent out via the I/O ports of the controller to the ferrite tuner power supplies and then the controller waits for the next computation cycle to begin. Because the main calculation is more than solving simple linear equations, the computation time can vary from cycle to cycle. Setting a long computation cycle would ensure all computations are done properly, but would also slow down the entire system and possibly become too slow for quick responses when needed. On the other hand, setting a fast computation cycle would render a number of unfinished computations (no change in the control voltages when these happen), again cause some non-responsiveness of the system, and equivalently slow down the system. The time 
length of the computation cycle is selected after comprehensive tests with simulated data and also then fine-tuned with many plasma shots. In our current setup, the D-port and E-port FFT systems share the same server and the same real-time code, and each computation cycle is chosen to be $0.4 \mathrm{~ms}$. On the other hand, because of the magnetic coils have large inductance, the coil currents cannot be changed instantaneously given the limited capability of the power supplies. As a result, the tuning speed of the entire FFT system is mostly determined by the response time (in the order of ms) of the coil currents.

The FFT real-time controlling system can be viewed as a complicated feedback system. The system would first seek a route to the target, and then lock on the target. After detecting a small deviation from the matching, the controller would generate a response so as to move the matching toward the target within $0.4 \mathrm{~ms}$. When the control system was initially brought online, oscillations were sometimes observed. After a careful study, we determined that these oscillations were due to the errors involving the difference of the actual electrical lengths and the geometrical lengths of the transmission line components. Because of the errors, the solution for the perfect match can be off-target and out of the feedback bandwidth (note that it is a complex two-dimensional problem). The oscillations were only eliminated by carefully adjusting some of the geometrical parameters in the computation, for example, the electrical length of elbows and T-connectors. In the initial operation of the prototypical system reported in Ref. [11 ], a digital PID (proportional-integral-derivative) scheme was incorporated in the real-time control program in order to avoid any possible oscillations. This scheme was later by-passed because the time constant in the coil current ( 4 ms from -150 A to +150 A) made the feedback almost always over-damped. After the computation error mentioned above was minimized, the feedback system became very robust. One more consideration to improve the robustness of the feedback is to 
allow a threshold of being "good-enough": as long as $\left|\Gamma_{\mathrm{DC} 1}\right|<0.1$ (power reflection $<1 \%$ ), the algorithm would not try to calculate better solutions, and leave the existing settings alone. One other reason behind this $1 \%$ threshold is that the antenna loading is subject to continuous fast time scale fluctuations induced by plasma. Further improvement to below $1 \%$ reflected power is impractical and it is also unnecessary. The FFT system has been running successfully and robustly for the 2014 campaign, and its performance is discussed in the next section.

\section{Performance of the FFT system on the D-port and E-port antennas}

The performance of the ferrite tuning systems is shown in an example plasma discharge in Figure 5. At $t=0.6 \mathrm{~s}$, the RF power levels, shown in panel-(d) and panel-(h), are detected to be above the pre-set threshold (30 kW), and then the stub lengths, shown in panel-(b) and panel-(f), are calculated. The control voltages for better matching, shown in panel-(c) and panel-(g), are calculated and then sent to the power supplies. Each volt in the control voltage drives $\sim 80$ Ampere current output from the power supplies, which are limited to be -150 A to $150 \mathrm{~A}$. After $2 \mathrm{~ms}$, the RF power reflection coefficients, $\left|\Gamma_{\mathrm{DC} 1}\right|^{2}$, of both antennas are reduced to less than $2 \%$ following the action of the FFTs. $\left|\Gamma_{\mathrm{DC} 1}\right|^{2}<1 \%$ is achieved after $5 \mathrm{~ms}$ and this low level of reflection is then maintained. Note that the traces for reflected power, shown in (d) and (h), are $10 \times$ magnified. Only after (and as soon as) the system detects a change in antenna loading that is large enough to cause $\left|\Gamma_{\mathrm{DC} 1}\right|^{2}$ to wander out of the $1 \%$ threshold, the feedback will then again try to adjust the coil currents. As a result, there are step-function-like features in the control voltages, panel-(c) and panel-(g) in the figure. As shown in panel-(d) and panel-(h), the reflected power as seen by the transmitters have been maintained within $\left|\Gamma_{\mathrm{DC} 1}\right|^{2} \leq 1.5 \%$ throughout the plasma 
discharge while the plasma confinement changes from L-mode, I-mode $(0.85-1.35 \mathrm{sec})$ and $\mathrm{H}$ mode $(1.35-1.5 \mathrm{sec})$. The $\mathrm{H}$-mode in this discharge is the Enhanced $\mathrm{D}_{\alpha} \mathrm{H}$-mode [16] which has no edge localized modes (ELMs). There is also about $1.5 \mathrm{MW}$ from $\mathrm{J}$ port during the power flat top. The spike in the reflected power in D antenna at $0.77 \mathrm{sec}$ and the two power drops on $\mathrm{E}$ antenna before $0.8 \mathrm{sec}$ were due to impurity injections from undetermined sources. From panel(a) and panel-(e) in the figure, we can see that VSWR $=(1+|\Gamma|) /(1-|\Gamma|)$ in the transmission line is reduced from above 10 on the antenna side of Stub-3 to $\leq 1.3$ on the transmitter side of Stub-1. In this discharge, each of the D-port and E-port antennas is able to successfully deliver $\sim 1.5$ MW to plasma out of the maximum $2 \mathrm{MW}$ available power from each transmitter. 

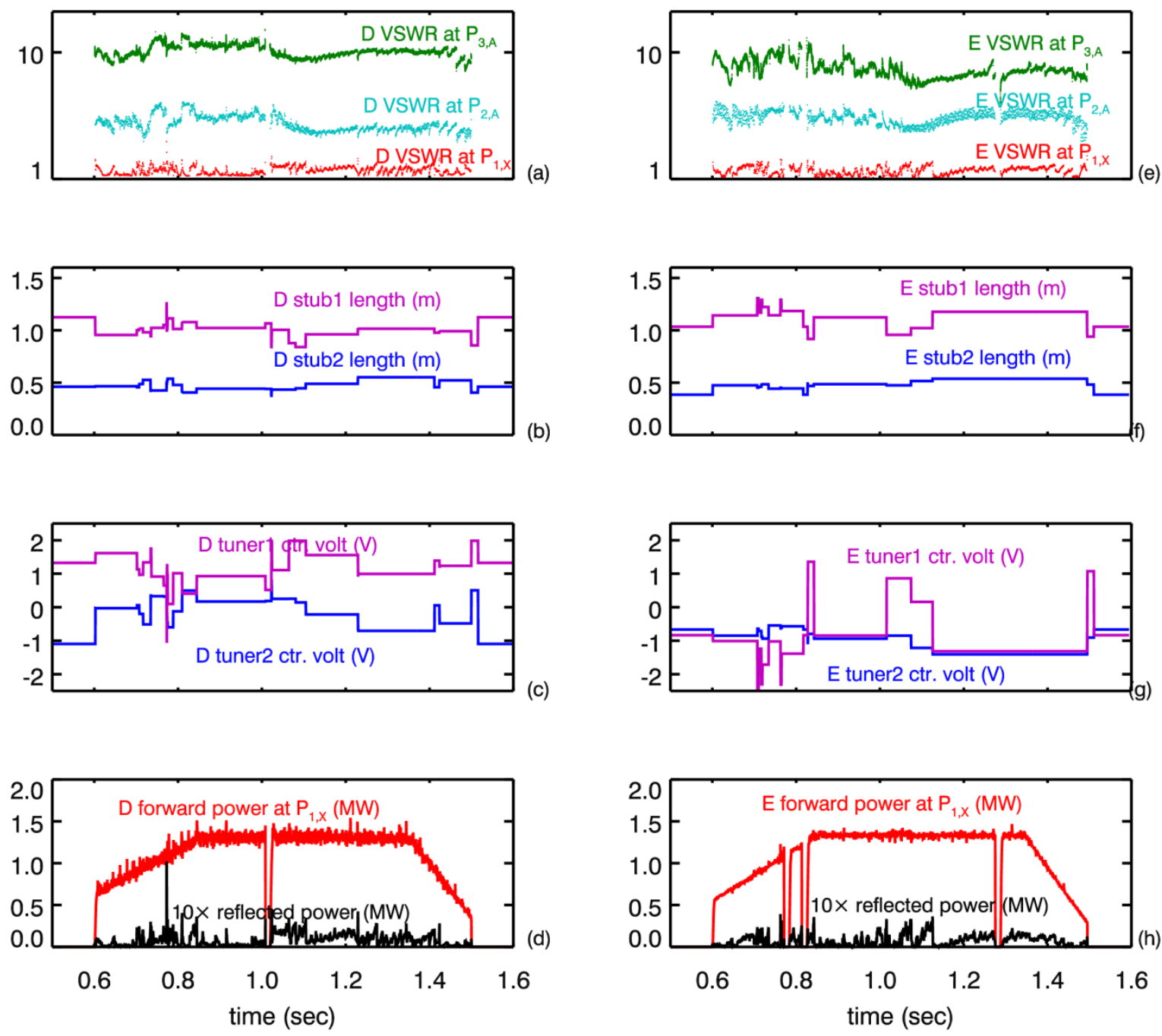

Figure 5 Time traces of a representative plasma shot. (a) and (e) are VSWRs (in logarithmic scale) at different locations shown in Figure 1 for the D-port antenna and E-port antenna respectively; (b) and (f) are the calculated stub lengths to achieve perfect match; (c) and (g) are the control voltages for the power supplies; and (d) and (e) are the forward and reflected power measured on the transmitter side of ferrite tuner \#1. The reflected power traces are magnified by $\times 10$.

Most H-mode discharges in Alcator C-Mod are Enhanced $\mathrm{D}_{\alpha} \mathrm{H}$-mode with no ELMs, and there are not many ELMy H-mode discharges. In Figure 6, we show the response of the FFT system to an ELM. The rise time of the ELM is about $0.5 \mathrm{~ms}$, and the decay time is several $\mathrm{ms}$. The FFT only detects the ELM after it has risen, and then starts to change the tuner control voltages in response. As shown in the figure, in the entire ELM period the reflected power is up and down twice, but it is maintained at $\leq 10 \%$ of the forward power (VSWR $\leq 2)$ and the match is returned 
to below $1 \%$ in a few computations. As a result, although in general, the FFT system does not have sufficient speed to maintain perfect matching during ELMs, it still alleviates the impact of ELMs, and a load resilient network for the D and E-port antennas is unnecessary. On the other hand, the speed of the FFT system prevents it from matching to an arc.
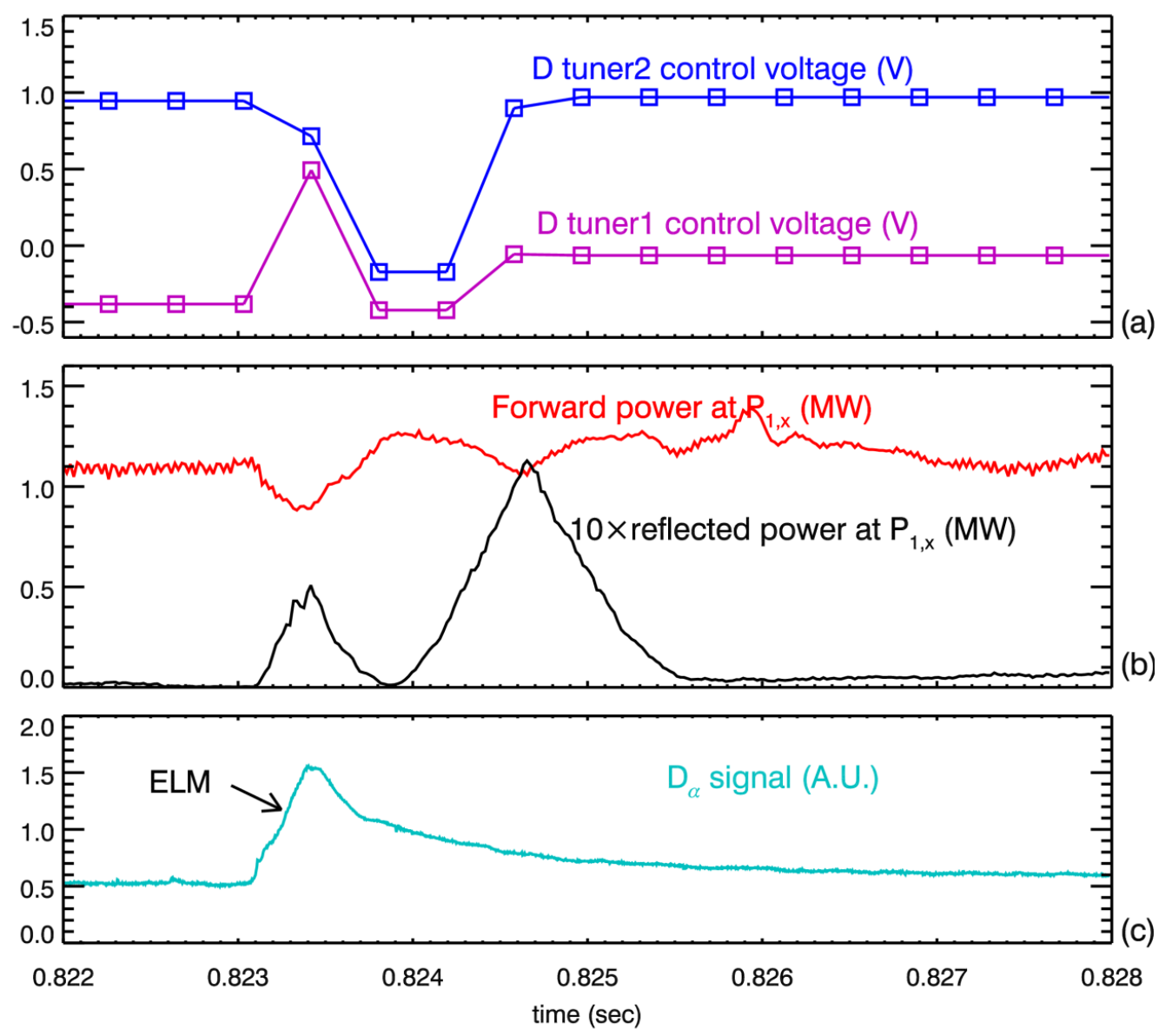

Figure 6 Time traces during an edge localized mode (ELM). (a). Control voltages for D tuners; (b)Forward and $10 \times$ reflected RF power traces; (c) edge $D_{\alpha}$ signal showing an ELM.

A second requirement for the FFT system is to minimize the maximum RF voltages (thus minimize the possibility of arcing) inside the ferrite tuners so that the tuners can operate reliably at high RF power. The maximum voltage in a transmission line section with characteristic 
impedance $\mathrm{Z}_{0}=50 \Omega$ is $\mathrm{V}_{\max }(\mathrm{kV})=\left[100 \times \mathrm{P}_{\text {net }}(\mathrm{MW}) \times \mathrm{VSWR}\right]^{1 / 2}$, and we can calculate the local standing-wave voltage at each point $\mathrm{P}, \mathrm{V}_{\mathrm{p}}=\mathrm{V}_{\max } \times\left|1+\Gamma_{\mathrm{p}}\right| /\left(1+\left|\Gamma_{\mathrm{p}}\right|\right)$, where $\Gamma_{\mathrm{p}}$ is the complex local voltage reflection coefficient. $\Gamma_{\mathrm{p}}$ at a point $\mathrm{P}$ that is distance $\mathrm{L}$ on the antenna side away from a reference point $\mathrm{P}_{0}, \Gamma_{\mathrm{p}}=\Gamma_{\mathrm{p} 0} \exp (\mathrm{j} \beta \mathrm{L})$, where $\beta=2 \pi / \lambda$ and $\lambda$ is the wavelength in vacuum. The voltage pattern inside a stub can be inferred using the Kirchhoff law at the connection point at the main transmission line.

In Figure 7, we show the calculated standing wave pattern of the FFT systems and transmission lines for $1 \mathrm{MW}$ net input RF power for the D-port antenna and E-port antenna for the cases that can be matched with zero coil current in all tuners. In panel-(a) and panel-(c), the voltages on the main transmission lines for D-port and E port are plotted respectively. The lengths of the transmission lines shown in the figure are real lengths in meter, relative to the location of Stub- 2 . The stub and tuner locations on the main line are indicated. The voltage in the transmission line sections are reduced from over $30 \mathrm{kV}$ on the antenna side of Stub-3 to $\sim 10 \mathrm{kV}$ on the transmitter side of Stub-1 (Note that $10 \mathrm{kV}$ is the theoretically lowest voltage for $1 \mathrm{MW}$ net RF power at VSWR = 1). The voltage inside a stub is determined by the length of the stub and the voltage at the T-connection to the main line, where the stub shares the same voltage as the main transmission line. In panel-(b) and panel-(d), the voltages inside the three stubs for D-port and E-port systems are plotted respectively. For the ferrite tuners, the horizontal axis is the equivalent electrical length. The maximum voltages inside the ferrite tuners are all below $20 \mathrm{kV}$ for $1 \mathrm{MW}$ net power on both systems. The highest voltage resides inside Stub-3 of the D antenna ( $38 \mathrm{kV}$ at $1 \mathrm{MW}$ ), and we have made sure that the particular location does not have any Teflon support, which would be susceptible to arcing at this high voltage. Note that the location and length of Stub-3 on both D-port and E-port antennas have been iterated several times in order to optimize 
the regime on the Smith chart that the FFTs can match and also minimize the voltage inside the ferrite tuners. With the current setup, even when we run $2 \mathrm{MW}$ (the limit of the transmitter) net power, the max voltage inside the tuners would still be less than $30 \mathrm{kV}$, well below the design voltage handling capability $(\sim 40 \mathrm{kV})$ of the tuners when filled with $\mathrm{SF}_{6}$.
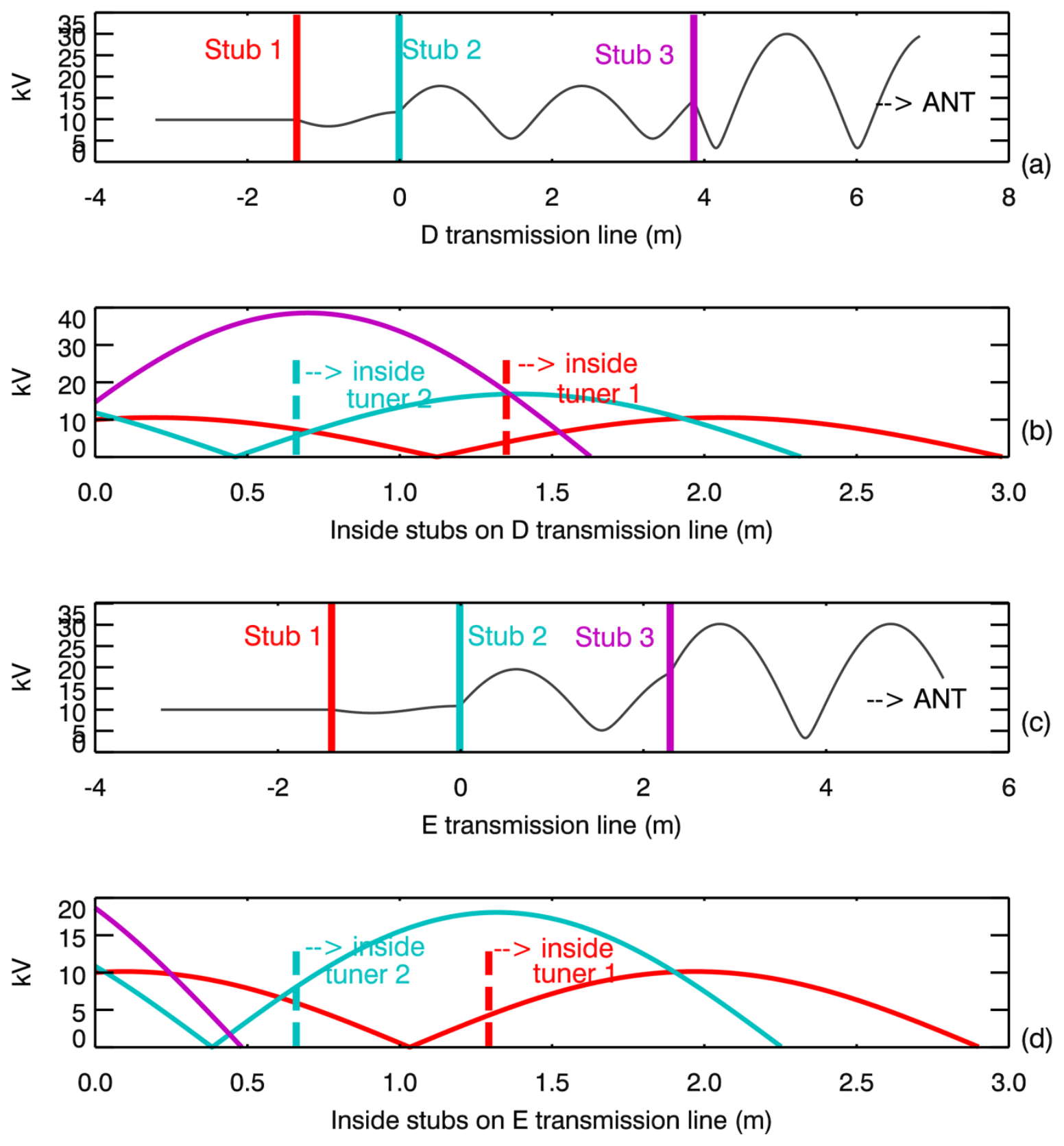
Figure 7 voltage patterns in the transmission lines and stubs for net RF power of $1 \mathrm{MW}$ for a typical case. Panel-(a) and panel-(b) are for the D-port antenna, while (c) and (d) are for the E-port antenna. Panel-(a) and panel-(c) are for the main transmission line while (b) and (d) are for the stubs. Note Stub-1 and Stub-2 each includes a normal transmission line section and a ferrite tuner.

Plasma current and density are the two main parameters that affect the antenna loading, and both act via changing the edge electron density profiles in front of the antenna.[17] As a result, the antenna loading varies not only within a plasma discharge, but it also varies discharge by discharge for different plasma parameters. In many tokamaks, the cutoff layer of the fast-wave is inside the last closed flux surface (LCFS), thus the distance from the antenna to the LCFS and the density profile inside the LCFS both play crucial roles in determining the antenna loading.[18] The situation on Alcator C-Mod is generally different because the edge electron density is typically much higher than that in other tokamaks. The fast wave cutoff layer in most plasma discharges is located inside the scrape-off-layer (SOL), and the distance to the antennas is insensitive to the edge density because of the steep density gradient in SOL. For the D-port and E-port antennas and the former 4-strap J-port antenna [8,9], the steepness of the edge density profile does affect the loading quite significantly: steep density pedestal in H-mode plasmas reduces the loading compared to that in L-mode plasmas.[17] If we populate the measured $\Gamma$ of all types of plasmas on a Smith chart, the complex $\Gamma$ from a fixed location of the unmatched transmission line usually form a cluster with a spread in both magnitude and phase of $\Gamma$, indicating that the loading variation has both resistive and capacitive (or inductive) components. An ideal real-time matching system must be able to handle the entire parameter space.

In Figure 8 and Figure 9 we show the performance of the FFT systems over the 2014 experimental campaign including all run-days when the FFT systems were operating and 
selecting the discharges where the net antenna power is greater $200 \mathrm{~kW}$. Each discharge is represented by $\sim 50$ data points on the figures: there are total $\sim 500$ shots for the D-port antenna and $\sim 200$ shots for the E-port antenna. The clusters of $\Gamma$ at different locations on the transmission lines are shown with different colors on the Smith charts. The cluster at the highest VSWR (in green) is $\Gamma$ from the unmatched section between Stub-3 and the antenna, measured by directional coupler DC3 shown in Figure 1. The shape by enclosed by green curves indicates a regime that can be transformed to the target of zero reflection by the FFT system. The cluster of blue points represents $\Gamma$ on the transmitter side of Stub-3, while the brown points represent $\Gamma$ on the antenna side of Stub-2. The trajectory of the transformation is represented by $\mathrm{P}_{3, \mathrm{~A}} \rightarrow \mathrm{P}_{3, \mathrm{X}} \rightarrow$ $\mathrm{P}_{2, \mathrm{~A}}$ in these figures. The cluster of red points represents $\Gamma$ on the transmitter side of Stub-1, which determines the reflection coefficient at the transmitters. The path of transformation from $\mathrm{P}_{2, \mathrm{~A}}$ to $\mathrm{P}_{1, \mathrm{X}}$ via the tuners has been shown previously in Figure 3. Note that almost all the points in the red cluster in both Figure 8 and Figure 9 have $|\Gamma| \leq 0.1$, corresponding to the power reflection coefficient $|\Gamma|^{2} \leq 1 \%$. The few points that are outside this region are points either during fast plasma transitions or in the beginning of the RF pulse when the FFTs need to seek and lock onto the target through its real-time algorithm. 


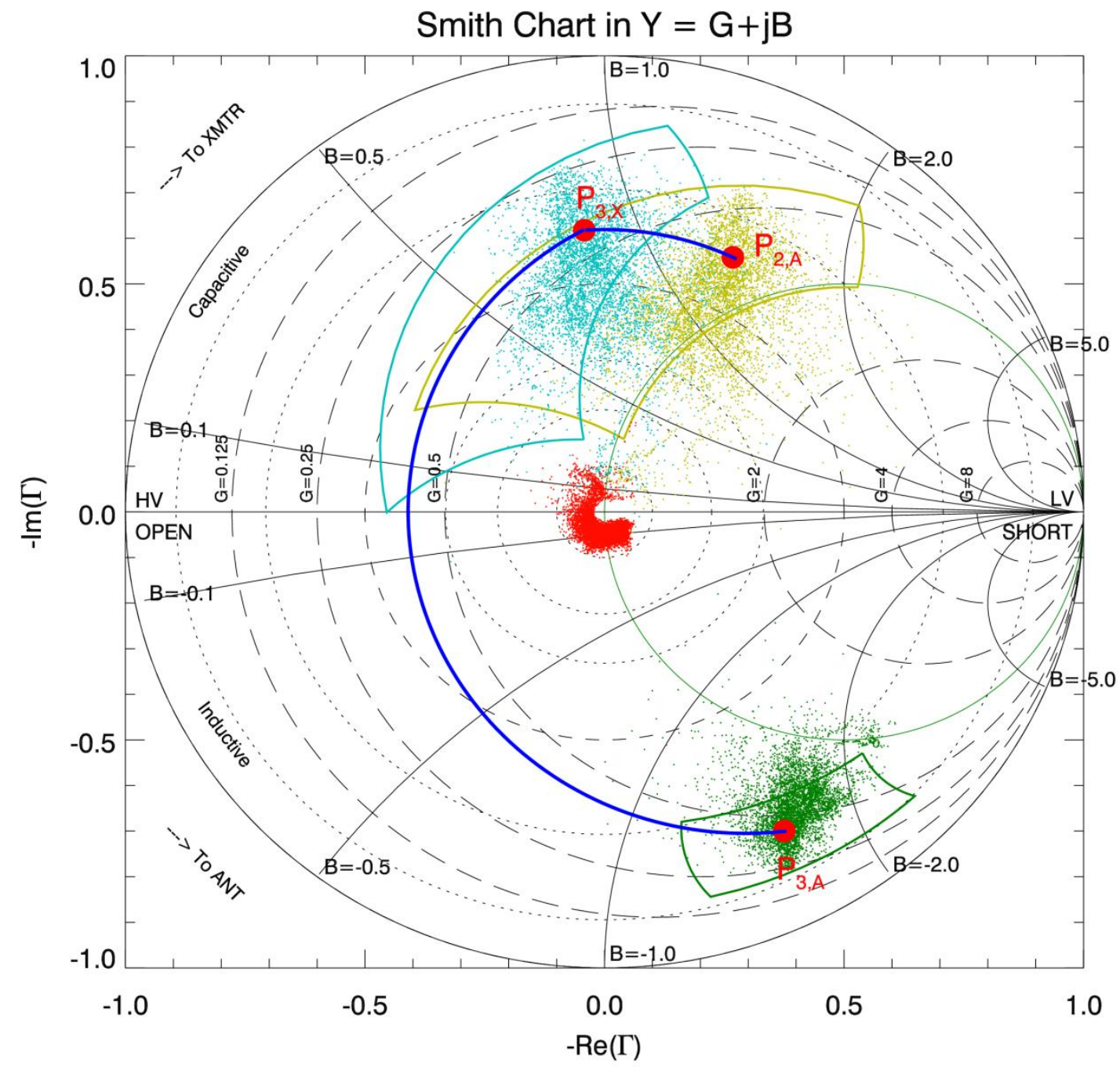

Figure 8 Performance of the ferrite tuning system on the D-port antenna over 2014 experimental campaign. Locations of the representative points are shown in Figure 1. 


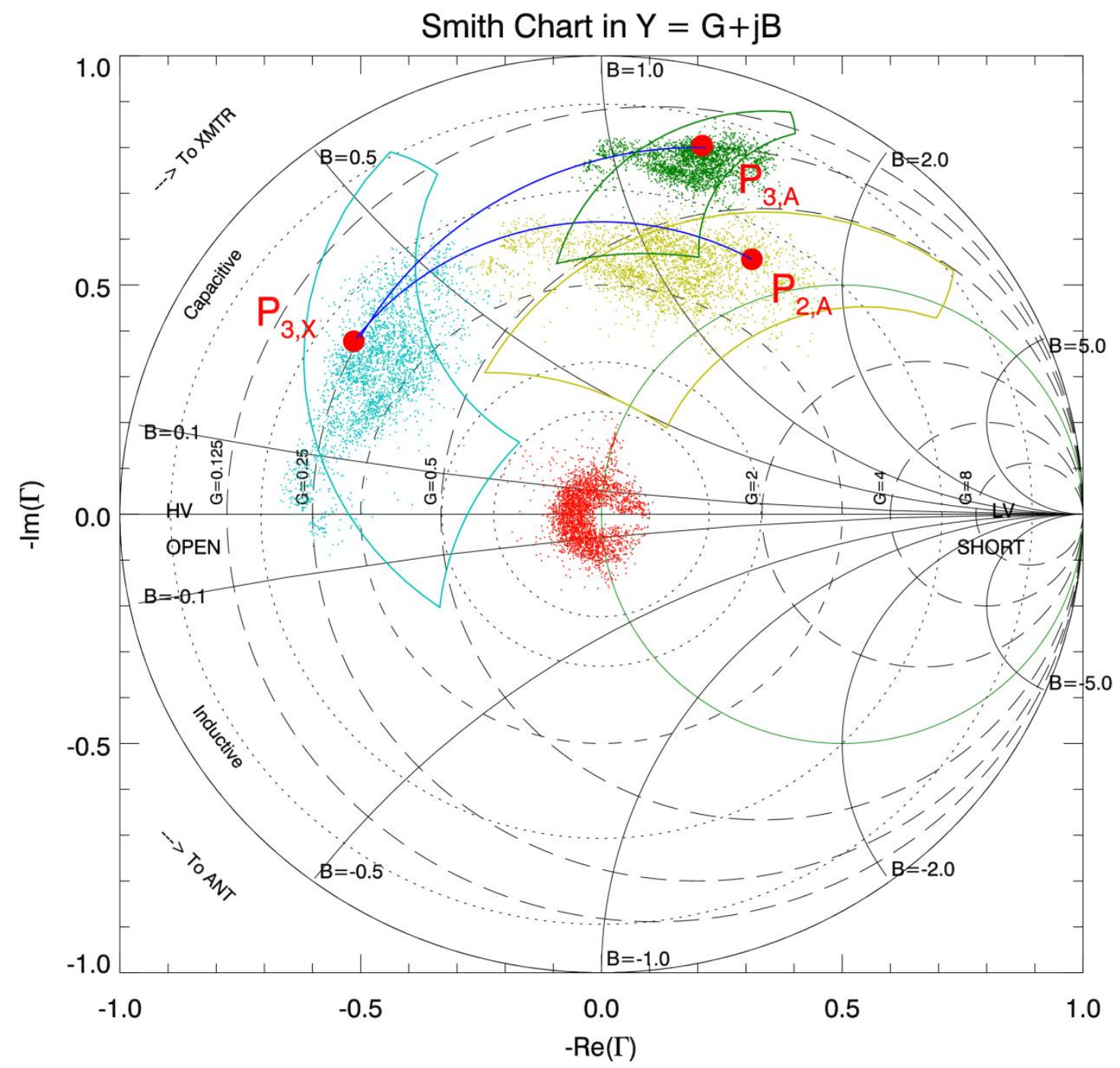

Figure 9 Performance of the ferrite tuning system on the E-port antenna over the $\mathbf{2 0 1 4}$ experimental campaign. Locations of the representative points are shown in Figure 1.

Ferrite tiles are not lossless for RF applications. There are generally two kinds of power loss mechanisms, dubbed as dynamic loss, which can happen at any RF power level, and nonlinear loss, which only happens at very high RF power intensity.[19] In the previous study [4], we found the evidence of non-linear power loss in the ferrite tuners during high power operation 
during the first implementation of the tuners without Stub-3, where the circulating RF power inside the tuners was very high. After several iterations of optimization, especially with the careful design and installation of Stub-3, the circulating power in the ferrite tuners at the same net power to the antennas has been significantly lowered. As a result, nonlinear power losses at high RF power intensity have not been observed in the 2014 campaign. However, the dynamic loss, due to re-crystallization of the ferrite material under oscillating RF magnetic field, still exists. This effect would be stronger when the bias magnetic field pushes the ferrite into the regime that the magnetic hysteresis loop in the magnetization curve becomes non-negligible. By comparing the net RF power as measured on the antenna side and the net power measured on the transmitter side of the FFT system, we believe that the dynamic loss is indeed a potential problem for the ferrite application under some conditions. In Figure 10, we show the power loss to the tuners vs. the bias coil current of one of the tuners for the E-port system for all the plasma discharges in the 2014 campaign. The power loss is calculated from the difference in the RF net power measured by directional couplers DC1 and DC2, as shown in Figure 1. There is no directional coupler between the two tuners; therefore the power loss is approximately the total loss in the two tuners. In Figure 10, a trend is revealed that toward the negative coil current (down to -150 A) of ferrite Tuner 1, presumably at the bias magnetic field is farther away from the ideal operational regime, the RF power losses increase quite significantly (up to 6\% ). The coil current of Tuner 2 typically varies within a much smaller range near zero coil current, and no correlation with the power losses has been found. A similar trend also exists on the D-port FFT system that power losses are observed when the tuners are biased at large negative coil currents. At these biasing currents, the ferrite tiles may be operating in the regime of the magnetization curve where hysteresis is not negligible. This observation suggests that the 
operational parameter space of the ferrite tuners needs to be carefully considered and the cooling capacity for the ferrite tiles may become a crucial limitation if the tuners are to be used in long pulse plasma discharges with high ICRF heating power. For some very high power operation, we may have to sacrifice some matching performance by limiting the coil currents to a narrower range where the ferrite tiles are operating in the low loss regime on the magnetization curve.

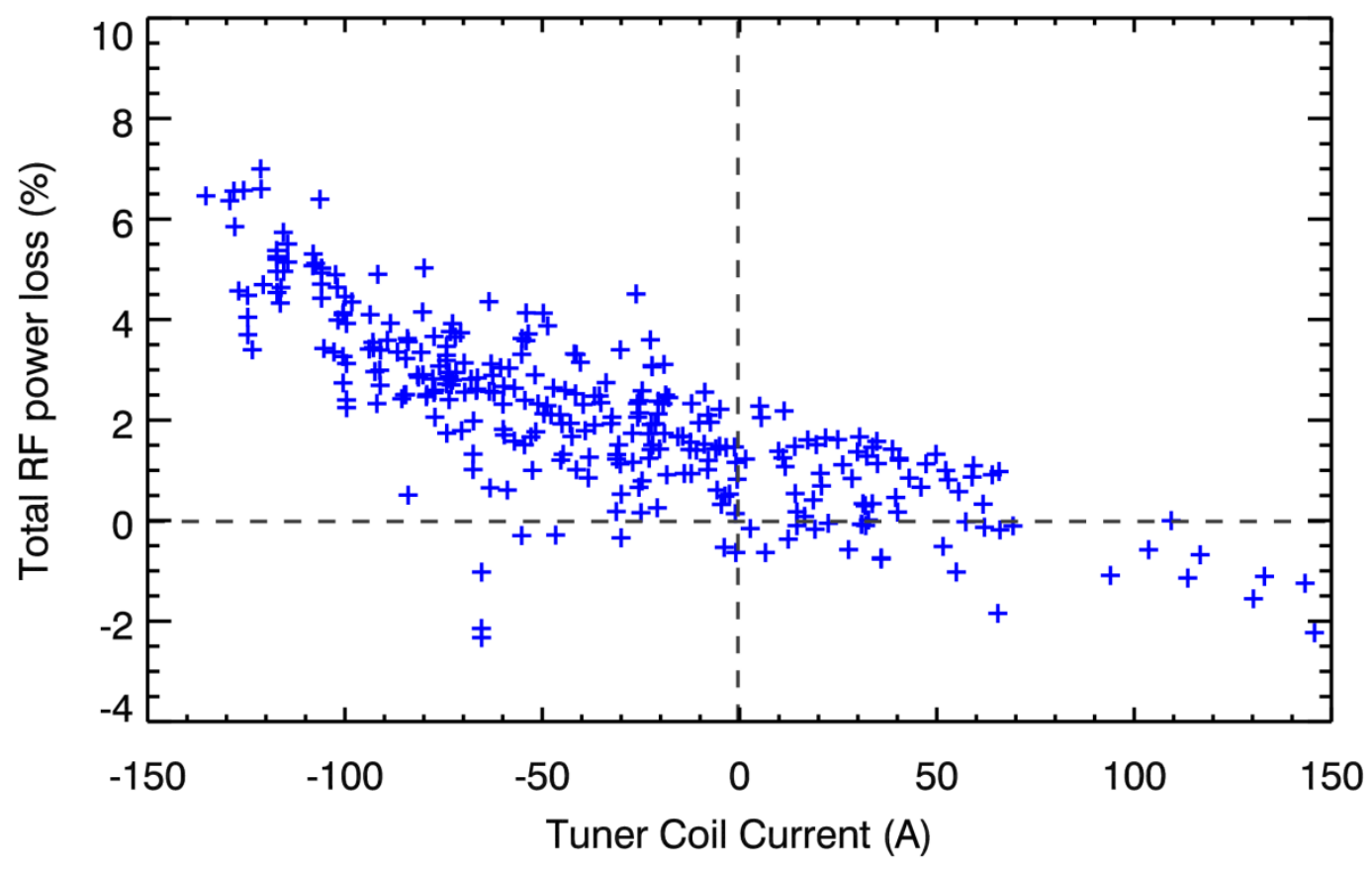

Figure 10 RF power loss (from DC2 and DC1 shown in Figure 1) correlated with the coil current of ferrite tuner 1 for the E-port antenna.

\section{Antenna matching on the field-aligned J antenna}

The antenna currently installed on the $\mathbf{J}$ port is an innovative field aligned (FA) 4-strap antenna.[10] The antenna straps are rotated to be perpendicular to the magnetic field lines near 
the edge (designed for the most common plasmas on Alcator C-Mod with $\mathrm{B}_{\mathrm{t} 0}=5.4 \mathrm{~T}$ and $\mathrm{I}_{\mathrm{p}}=1$ MA). The antenna shape is also conformed to the typical plasma shape on Alcator C-Mod. Viewed along field lines in front of the antenna, the antenna structure is uniform and symmetric. After two experimental campaigns, the antenna has been shown to generate fewer impurities under similar conditions than the traditional D-port and E-port antennas and also better than the former 4-strap J-port antenna, where the antenna straps were vertical and the antenna structure was toroidal. In this Section we discuss the matching system for the FA J-port antenna.

A surprising observation of the FA antenna is the insensitivity of its antenna loading vs. plasma parameters. Although it has very similar configuration of power feeding and decoupling schemes as the former 4-strap antenna on J-port, the new antenna has a much smaller spread in antenna loading, as shown in the spread of $\Gamma$ in magnitude and phase measured on the unmatched transmission line. This is also true when comparing the FA J-port antenna with the D-port and Eport antennas in the same plasma discharge. In Figure 11, we show a comparison of the FA J antenna vs. the $\mathrm{E}$ antenna in an $\mathrm{H}$-mode plasma with edge localized modes (ELMs). The ELMs suddenly eject plasmas outward and create temporal spikes of the local electron density in front of the antennas. Because the fast wave cutoff layer is moved closer to the antennas with the increased local density, the loadings increase during ELMs. Shown in the measured data, ELMs would induce downward spikes in $|\Gamma|$ in the unmatched line, which suggests a decrease of VSWR and increased loading. Note that a decrease in $|\Gamma|$ in the unmatched line does not necessarily mean a better match seen by the transmitter. Figure 11 shows that the changes in both magnitude and phase of $\Gamma$ for the FA J-port antenna are much smaller compared to those of the E-port antenna. 


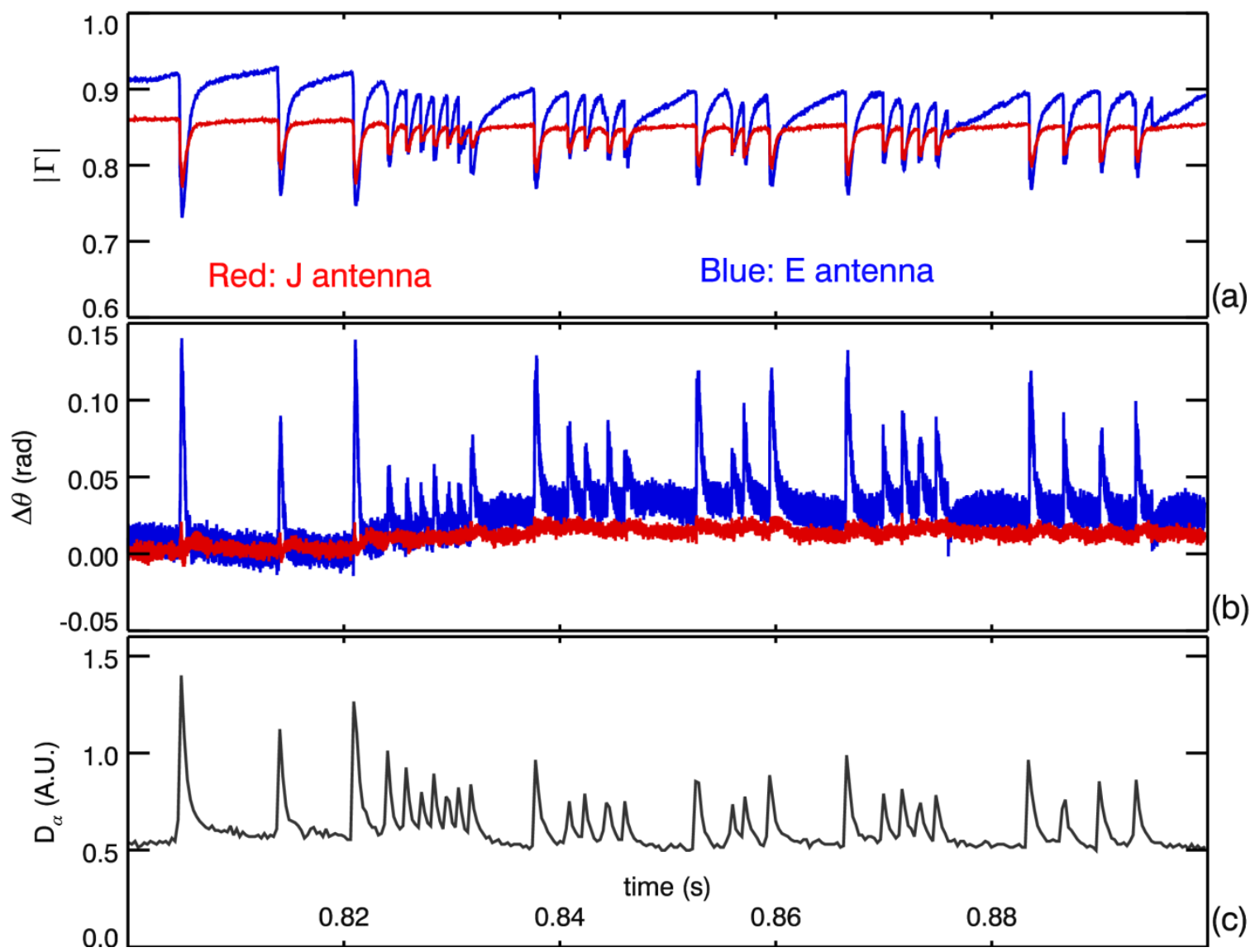

Figure 11 Comparison of antenna responses to ELMS for the FA J-port antenna (red traces) and the Eport antenna (blue traces) in the same plasma discharge. (a) the magnitude of reflection coefficient in the unmatched line; (b) phase; (c) $D_{\alpha}$ trace showing ELMs.

The physics behind this load insensitivity is beyond the scope of this paper. Some discussions can be found in Ref. [20]. The FA J-antenna is fed by two transmitters, and each transmitter is connected to 2 straps while there is decoupling loop separating these two transmission lines.

Therefore, the complex $\Gamma$ measured on the transmission line cannot be transformed to the antenna impedance in a simple way. A hypothesis is that because of the antenna alignment and symmetry along the field lines, some of the responses to the plasma among the antenna straps may cancel each other. In the coming experimental campaign, we plan to have more voltage and 
current measurement on the antenna and calculate the antenna impedance matrix. In this paper, we discuss how we successfully utilized the load-tolerance feature to devise a simple antenna matching network for the FA J-port antenna.

There are two transmitters for this 4-strap antenna, and the two transmission lines are labeled as J3 and J4. In the 2012 campaign when the FA J-port antenna was first installed and operated, we had the standard mechanical stub tuner and phase shifter in the transmission lines for antenna matching. When running high power, the mechanical stub tuners and phase-shifters became the weakest links in the transmission lines (because of high local RF voltage), and break-down occurred inside these components. In the recent 2014 campaign, we decided to add pre-match stubs on the system near the antenna (one stub for each line). The locations and lengths of these stubs are designed based on a large amount of available FA J-port antenna data. The stubs are added at the locations where the $G=1$ circle and the VSWR circle intersect on a Smith admittance chart. The lengths of the stubs are chosen to cancel the imaginary component $j \mathrm{~B}$ of the admittance $\mathrm{Y}$ on the antenna side of the stubs, and thus minimize $|\Gamma|$ on the transmitter side of the stub for most plasma shots. The installation of these stubs has greatly reduced the VSWR in the transmission line sections between this pre-match stub and the mechanic stub tuner and phase shifter. Figure 12 shows the effect of this installation. VSWR in the section of the line is reduced from average 8.9 before the installation to average 2.4 after the installation. This VSWR reduction is equivalent to a reduction of $\mathrm{V}_{\max }$ from $30 \mathrm{kV}$ to $16 \mathrm{kV}$ for $1 \mathrm{MW}$ net RF power. Because the pre-match stubs are installed close to the antenna, the voltages have been reduced on the main part of the transmission lines all the way back to the transmitter, including the mechanical stub tuners and phase shifters. Together with some minor adjustments on the mechanical stub and phase shifter between plasma discharges, quite reasonable matching can be 
obtained for most plasma discharges with reduced voltage and power handling issues. In high performance I-mode plasma discharges [21], where unlike in H-mode plasmas the plasma edge does not have a steep density pedestal, total 3.7 MW RF power has been successfully delivered by the FA J-port antenna alone from the two $2 \mathrm{MW}$ transmitters.

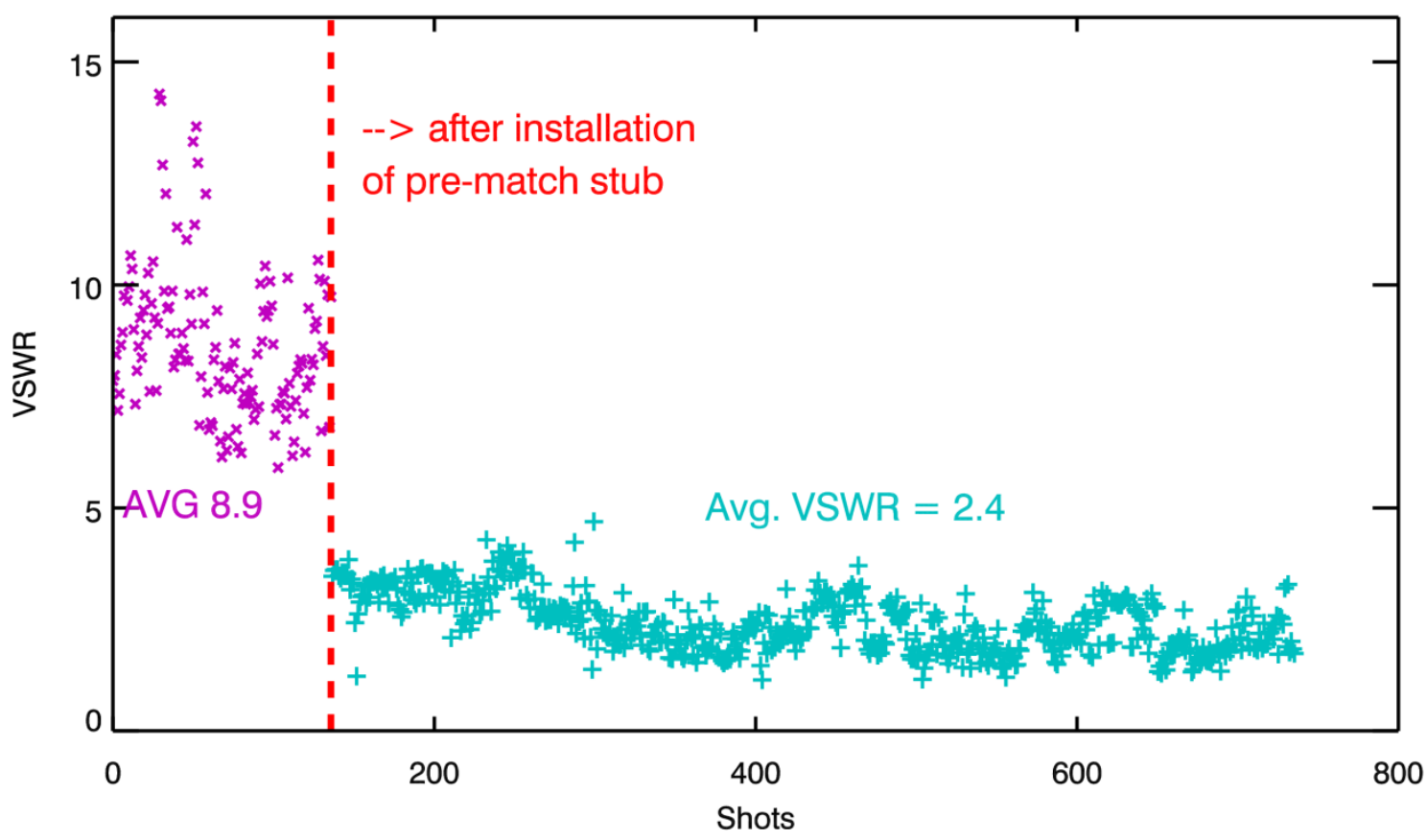

Figure 12 Effect of the pre-match stub on the VSWR of the J3 transmission line. X-axis is the plasma discharges in the $\mathbf{2 0 1 4}$ campaign in the order of the date and time when they were taken.

An idea to further utilize this feature of loading insensitivity is to install a real-time matching system using a single ferrite tuner plus a fixed stub. Here we show the feasibility of such a system. Note that a ferrite tuner can only change the susceptance B part of the admittance $\mathrm{Y}=\mathrm{G}$ $+j \mathrm{~B}$; therefore, we need to install this ferrite tuner near a location of $\mathrm{G}=1$ on a Smith chart. We also need to make sure that the spread of the data has the smallest spread in G. With this consideration, the ferrite tuner can then vary $\mathrm{B}$ to make $\mathrm{B} \rightarrow 0$ on the transmitter side. The 
spread of $\Gamma$ for a large number of different plasma discharges is somewhat larger than that shown in Figure 11 for a single plasma discharge. In Figure 13, we show the transformation process from $\Gamma$ on the unmatched transmission line section $\Gamma_{\mathrm{DC} 2}$ to the optimized match at $\mathrm{P}_{1, \mathrm{X}}$. Here $\mathrm{P}_{2, \mathrm{X}}$ is the location on the transmitter side of the pre-match stub. This pre-match stub would be at a different location and have different length than the one presently installed after the system optimization together the ferrite tuner. It rotates around the constant VSWR circle along a section of transmission line to the antenna side of the ferrite tuner, $\mathrm{P}_{1, \mathrm{~A}}$. The ferrite tuner then is able to transform $\mathrm{P}_{1, \mathrm{~A}}$ along a constant $\mathrm{G}$ circle to have minimal $|\Gamma|$ on the transmitter side of the tuner, $\mathrm{P}_{1, \mathrm{X}}$. Usually $\mathrm{P}_{1, \mathrm{X}}$ is a point on the horizontal line where $\operatorname{Im}(\Gamma)=0$ and $\mathrm{B}=0$ on the Smith chart. The optimization is done through computerized scan of stub parameters by minimizing the spread of $\mathrm{G}$, making all $\mathrm{P}_{1, \mathrm{~A}}$ points as close to the $\mathrm{G}=1$ circle as possible for all the discharges from the 2014 campaign. As shown in Figure 11, by carefully choosing the parameters, a single ferrite tuner with real-time control can achieve power reflection $\leq 1 \%$ under most plasma parameters for this FA J-port antenna. This design will be implemented in the coming Alcator CMod campaigns. 


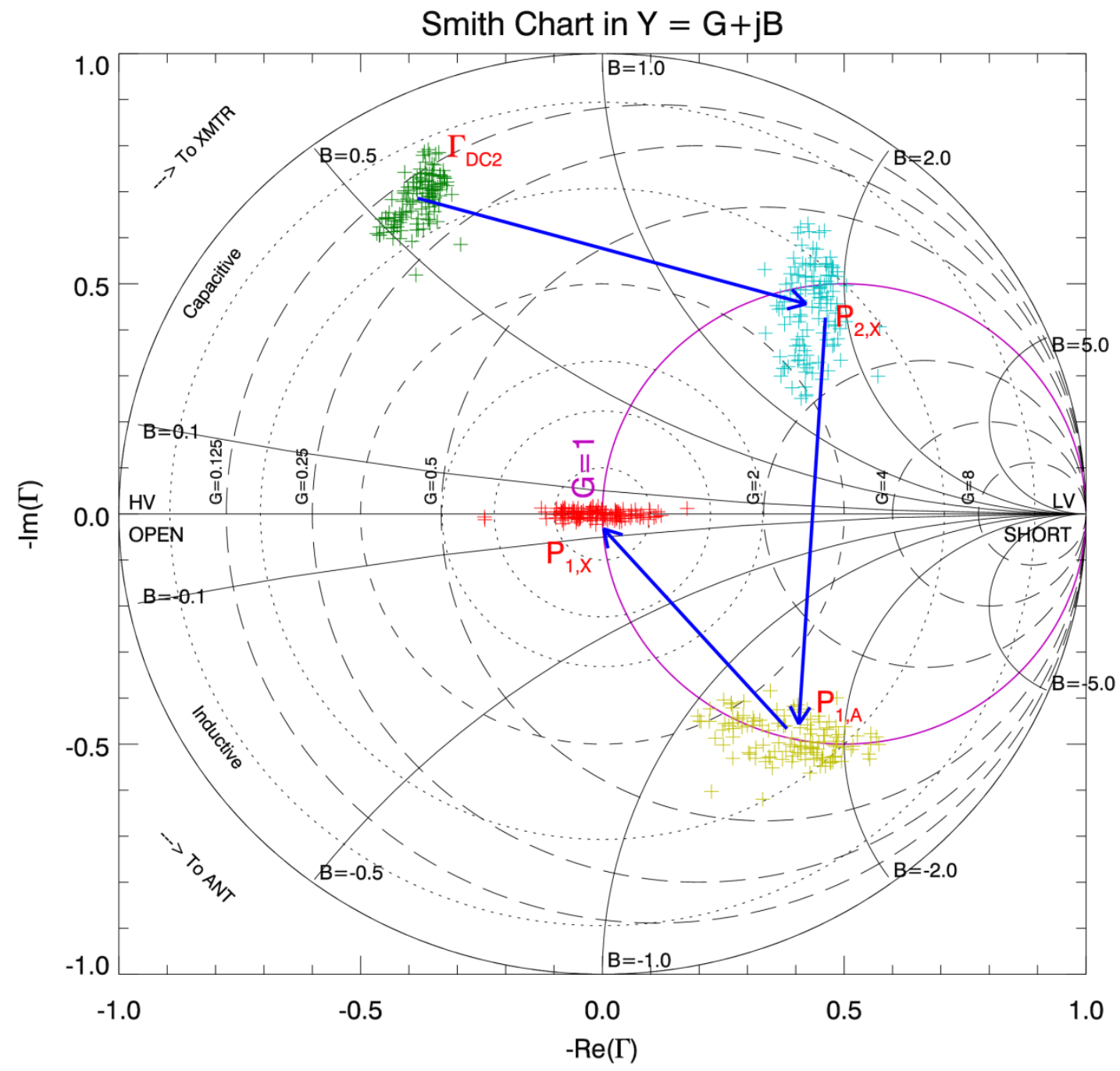

Figure 13 Design and optimization of $\mathrm{J}$ antenna matching using a single variable stub. $P_{2, x}$ is the point on the transmitter side of a fixed-length pre-match stub, $P_{1, A}$ is the point on the antenna side of the single ferrite tuner, and $P_{1, x}$ is on the transmitter side of the tuner. Optimization is based on all plasma shots in 2014 campaign.

\section{Summary}

Fast real-time antenna matching has been accomplished on the two traditional D-port and E-port ICRF antennas on the Alcator C-Mod tokamak using ferrite tuners. The system can achieve and maintain the antenna matching with power reflection below $1 \%$ for high RF power and under 
almost all plasma conditions. Pre-match stubs have been installed on the field-aligned J-port antenna and the medication has improved the reliability of the transmission lines and allowed high power operation of the FA J-port antenna. Design work has also shown that the matching of the FA J-port antenna can be further enhanced by adding a single ferrite tuner on each transmission line.

\section{Acknowledgements}

Work supported by US DoE Cooperative agreement DE-FC02-99ER54512 at MIT using the Alcator C-Mod tokamak, a DOE Office of Science user facility.

\section{References}

[1] A. Kaye, V. Bhatanagar, P. Crawley, C. Gormezano, J. Jacquinot, The JET wide-band matching system, in: P.M. Ryan, et al. (Eds), $12^{\text {th }}$ Topical Conference on Radio Frequency Power in Plasmas, AIP Conference Proceeding, vol. 403 (1997) 389.

[2] C.M. Qin, Y.P. Zhao, Y.Z. Zhao, J.Y. Din, P. Wang, Y.P. Pan, et al., Design of a new type of stub tuner in ICRF experiment, Plasma Sci. Technol. 3 (2003) 1779-1784.

[3] K. Saito, R. Kumazawa, T. Takahashi, M. Yokota, H. Takeuchi, T. Mutah, et al., Real-time impedance matching system using liquid stub tuners in ICRF heating, Fusion Eng. Design 81 (2006) 2837-2842.

[4] Y. Lin, A. Binus, S.J. Wukitch, Real-time fast ferrite ICRF tuning system on the Alcator CMod tokamak, Fusion Eng. Design 84 (2009) 33-37.

[5] G. Chen, Y. Zhao, Y. Mao, S. Yuan, G. Zheng, F. Zheng, et al., Design and Test of the Fast Ferrite Tuner for ICRF Heating in EAST, Fusion Sci. Tech. 61 (2012) 301. 
[6] S.J. Wang, B.G. Hong, Development of fast ferrite tuner for ICRF experiment in KSTAR tokamak, J. Kor. Phys. Soc. 49 (2006) S302-S304.

[7] E.S. Marmar, The Alcator C-Mod program, Fusion Sci. Technol. 51 (2007) 261.

[8] P.T. Bonoli, R. Parker, S.J. Wukitch, Y. Lin, M. Porkolab, J. C. Wright, et al., Wave-particle studies in the ion cyclotron and lower hybrid ranges of frequencies in Alcator C-Mod, Fusion Sci. Technol. 51 (2007) 401.

[9] S.J. Wukitch, R.L. Boivin, P.T. Bonoli, J.A. Goetz, J. Irby, I. Hutchinson, et al., Investigation of performance limiting phenomena in a variable phase ICRF antenna in Alcator CMod, Plasma Phys. Control. Fusion 46 (2004) 1479.

[10] S.J. Wukitch, M.L. Garrett, R. Ochoukov, J.L. Terry, A. Hubbard, B. LaBombard, et al., Characterization and performance of a field aligned ion cyclotron range of frequency antenna in Alcator C-Mod. Physics of Plasmas, 20 (2013) 056117.

[11] Y. Lin, J.A. Stillerman, A. Binus, A. Parisot, S. Wukitch, Digital real-time control for an ICRF fast ferrite tuning system on Alcator C-mod, Fusion Eng. Design 83 (2008) 241-244.

[12] S. Martin, W. Arnold, E. Pivit, A fast double mode tuner for antenna matching, in: D. Batchlor (Ed.), $9^{\text {th }}$ Top. Conference on RF Power in Plasmas, AIP conference Proceedings, Vol 244 (1992) 318-321.

[13] R. D'Inca, Arc detection for the ICRF system on ITER, in: C.K. Phillips and J.R. Wilson, (Eds), $19^{\text {th }}$ Top. Conference on Radio Frequency Power in Plasmas, AIP Conference Proceeding, vol. 1406 (2011) 5 .

[14] Philip H. Smith, Electronic Applications of the Smith Chart, SciTech Publishing (1995), Raleigh, NC, USA.

[15] J.A. Stillerman, M. Ferrara, T.W. Fredian, S. Wolfe, Digital real-time plasma control system for Alcator C-Mod, Fusion Eng. Design 81 (2006) 1905-1910.

[16 ] M. Greenwald, et al, Phys. Plasmas 6 (1999) 1943. 
[17] A. Parisot, S.J. Wukitch, P. T. Bonoli, J.W. Hughes, B. LaBombard, Y. Lin, R. Parker, M. Porkolab, A.K. Ram, ICRF loading studies on Alcator C-Mod, Plasma Phys. Control Fusion 46 (2004) 1781.

[18] M. J. Mayberry, S.C. Chiu, R.I. Pinsker, R. Prater, D. Hoffman , F.W. Baity, et al., Coupling of fast waves in the ion-cyclotron range of frequencies to H-mode plasmas in DIII-D, Nucl. Fusion 30 (1990) 579.

[19] J.E. Griffin, G. Nicholls, A review of some dynamic loss properties of Ni-Zn accelerator RF system ferrite, IEEE Trans. Nucl. Sci. NS-26(3) (1979) 3965.[

[20] S. Wukitch, D. Brunner, M.L. Garrett, et al "Evaluation of a field aligned ICRF antenna in Alcator C-Mod", $25^{\text {th }}$ IAEA Fusion Energy Conference, St. Petersburg, Russia, 13-18 October, 2014. Presentation FTP/1-1.

[21] D.G. Whyte, A.E. Hubbard, J.W. Hughes, B. Lipschultz, J.E. Rice, E.S. Marmar, et al., Imode: an H-mode energy confinement regime with L-mode particle transport in Alcator C-Mod, Nuclear Fusion, 50(2010), 105005. 


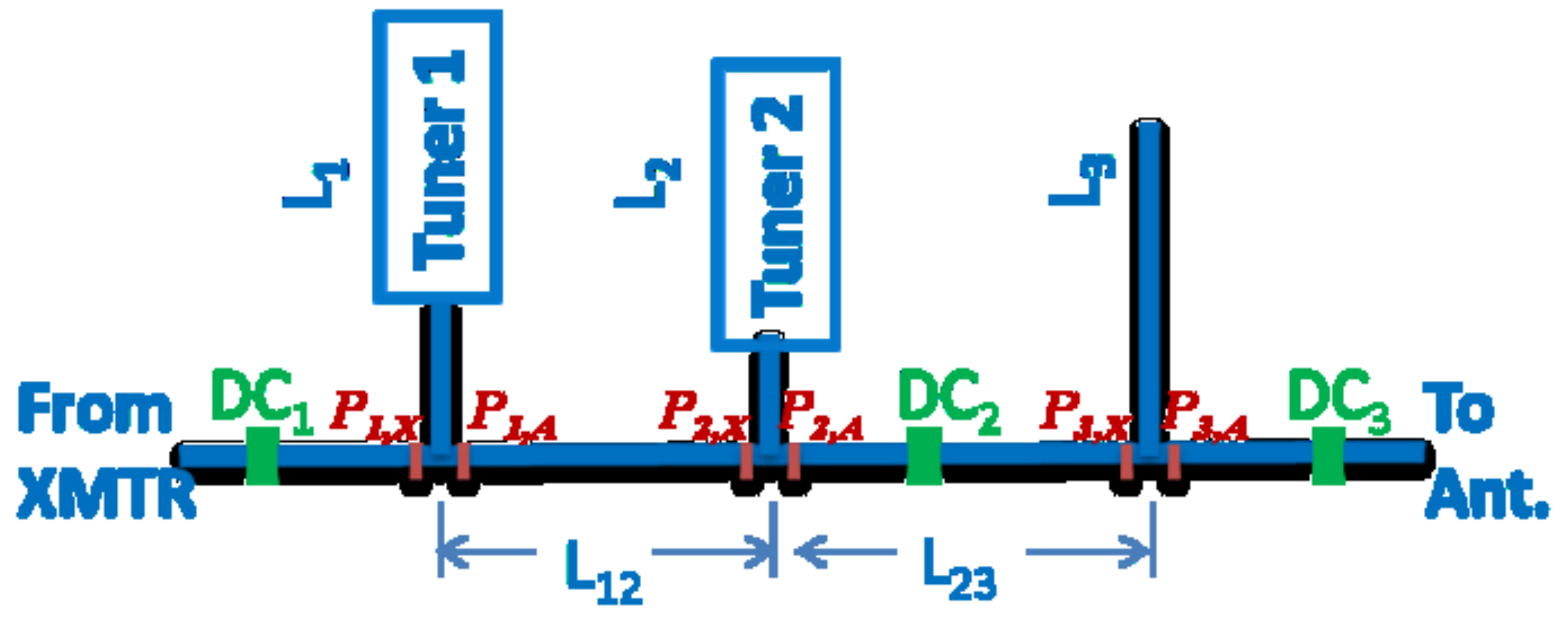




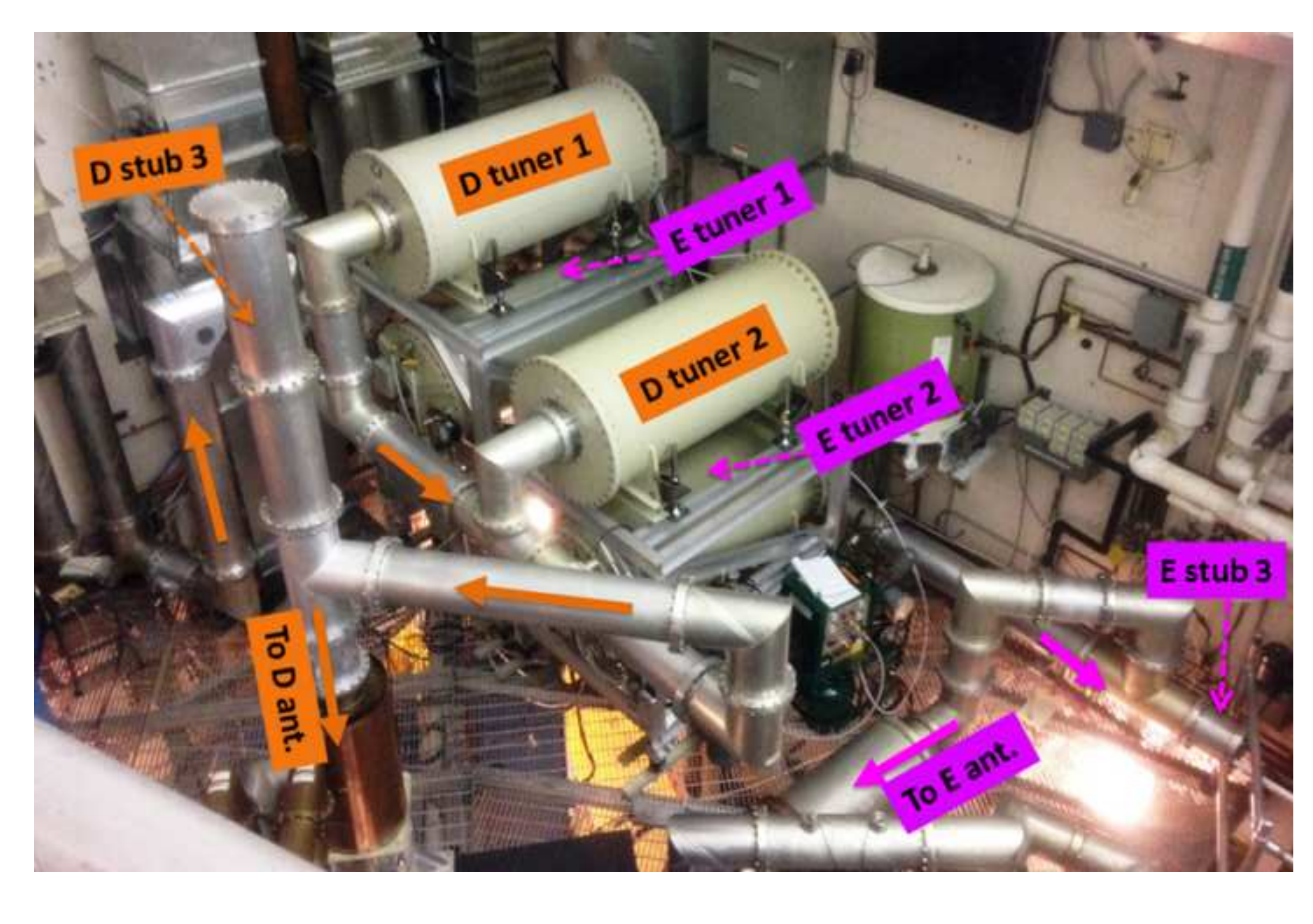

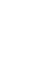

\author{
Fire
}
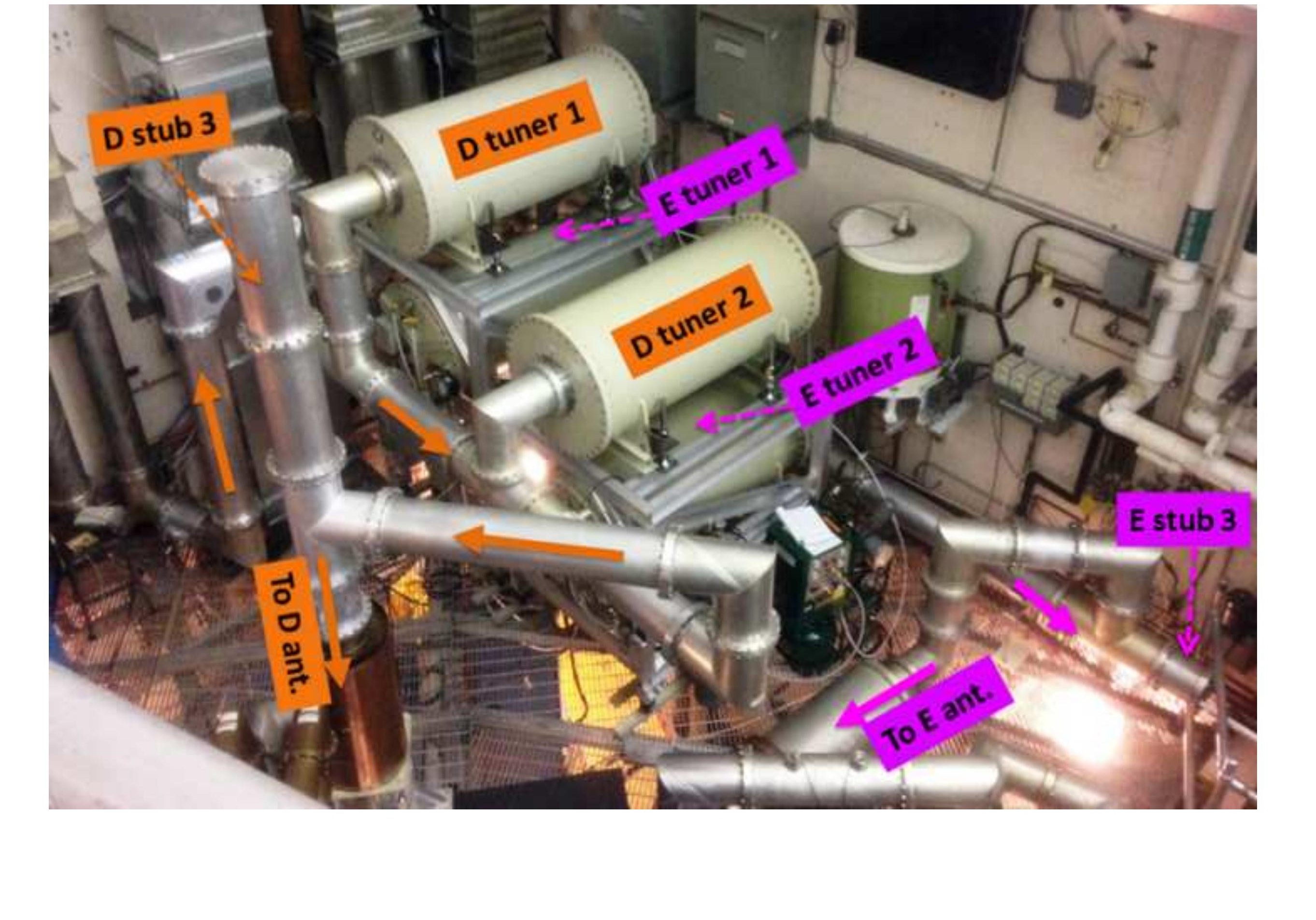


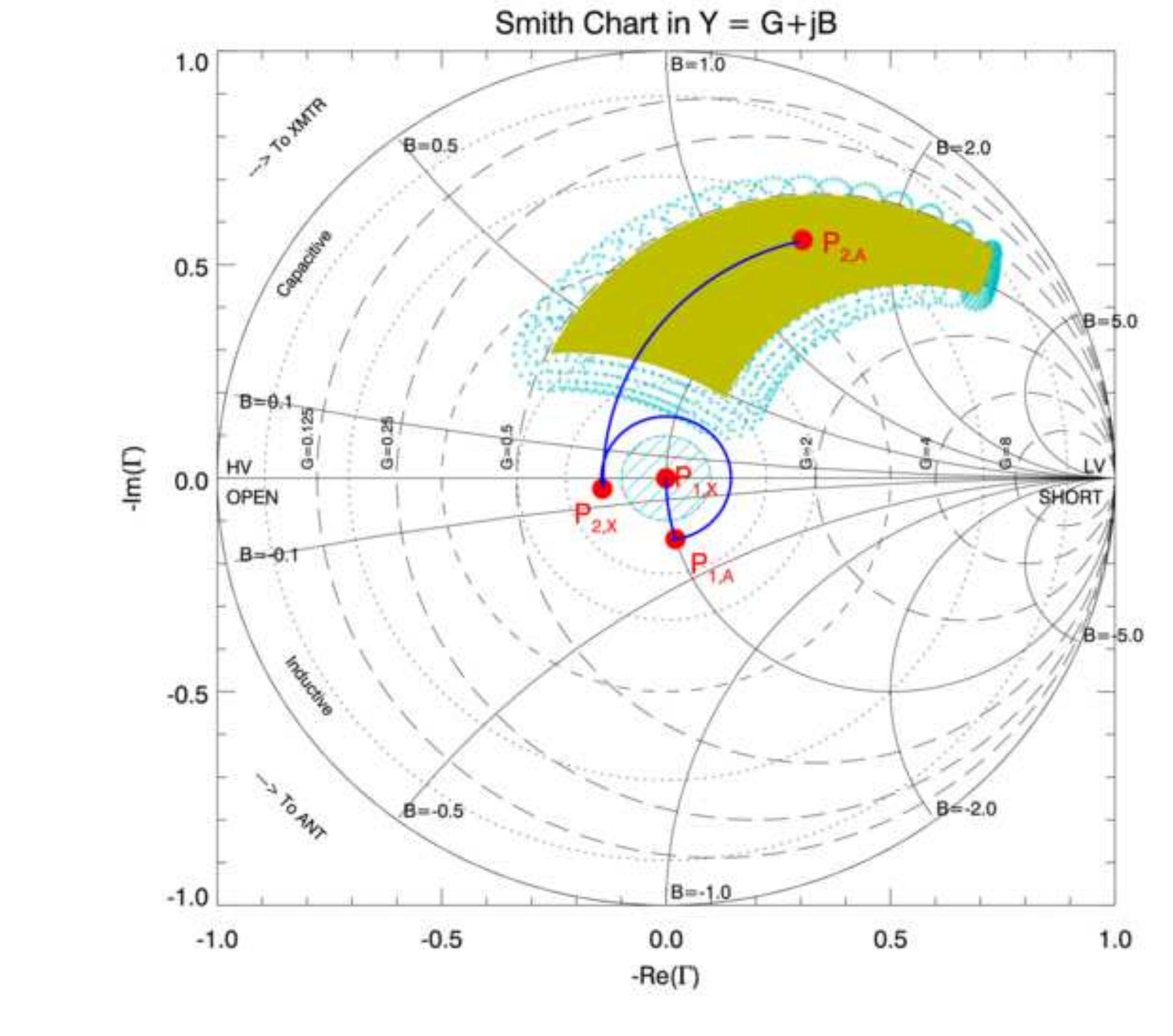

Smith Chart in $\mathrm{Y}=\mathrm{G}+\mathrm{jB}$

.

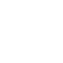

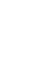
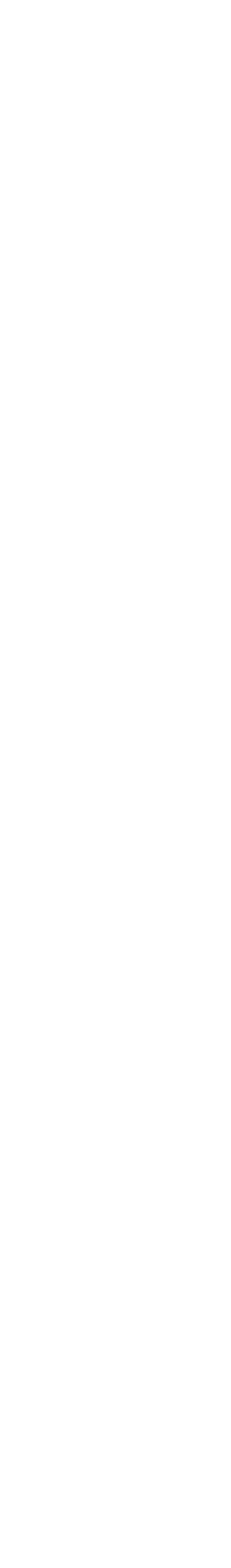

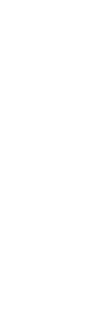




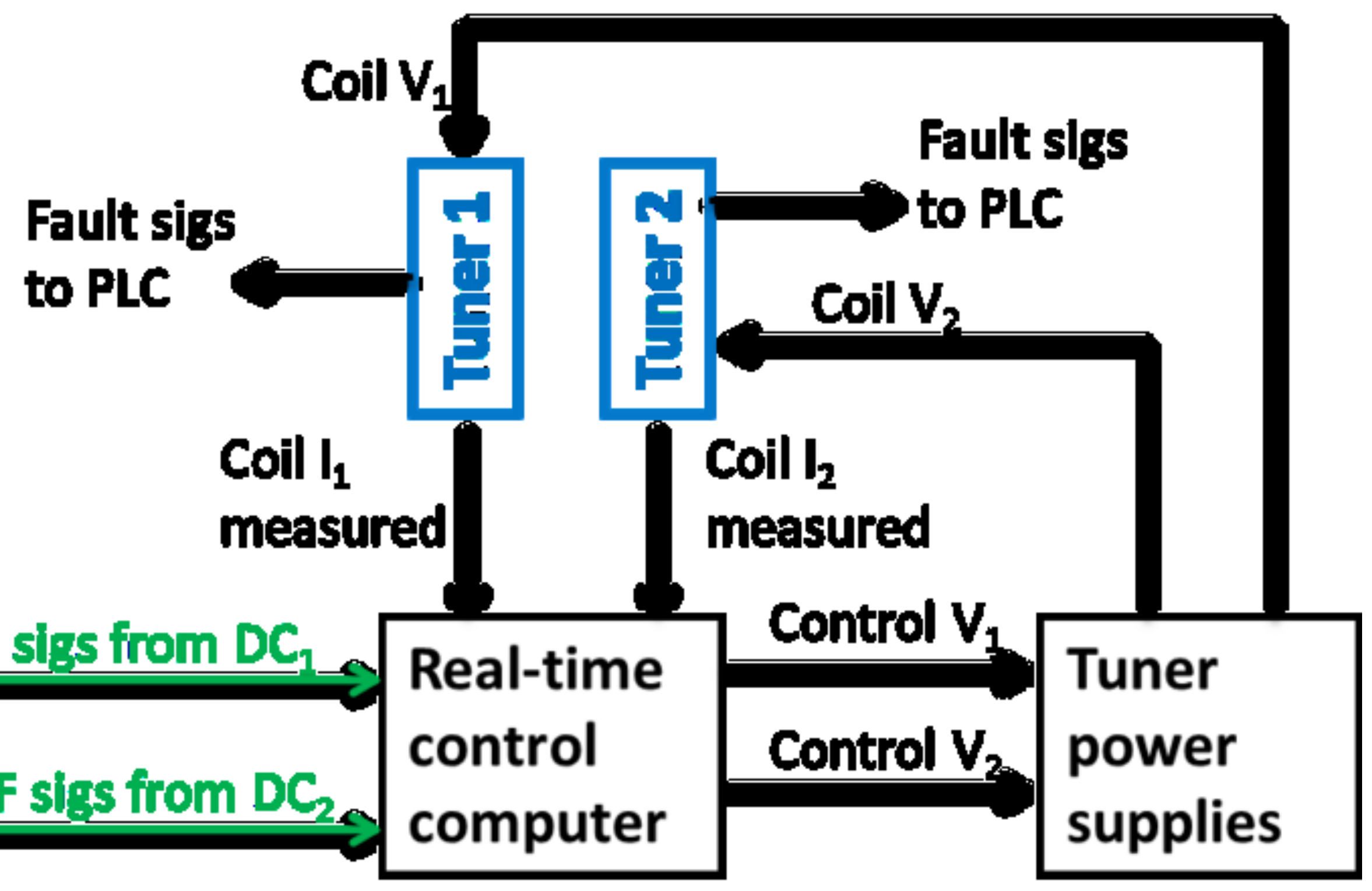




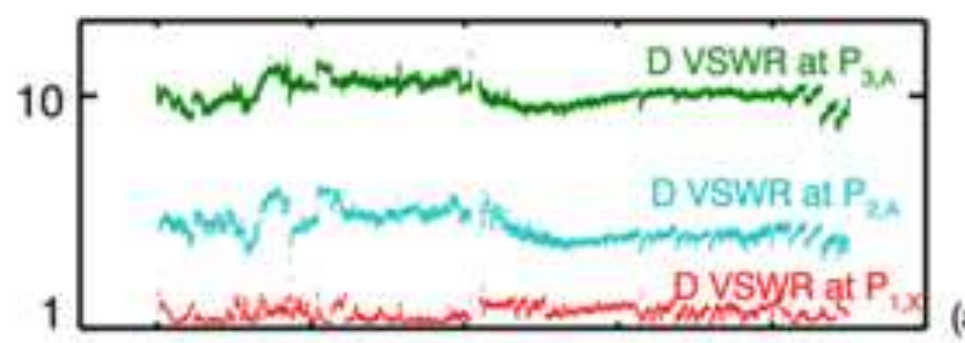

(a)

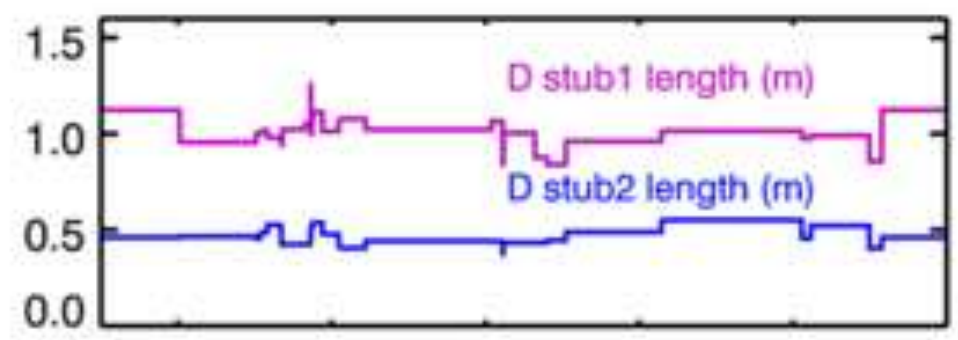

(b)

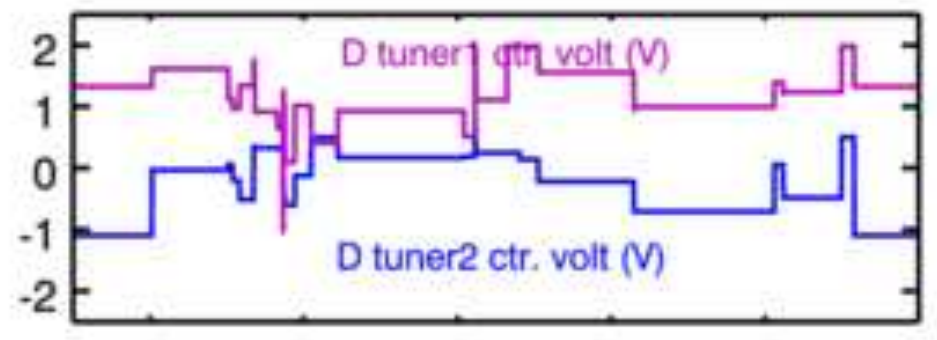

(c)

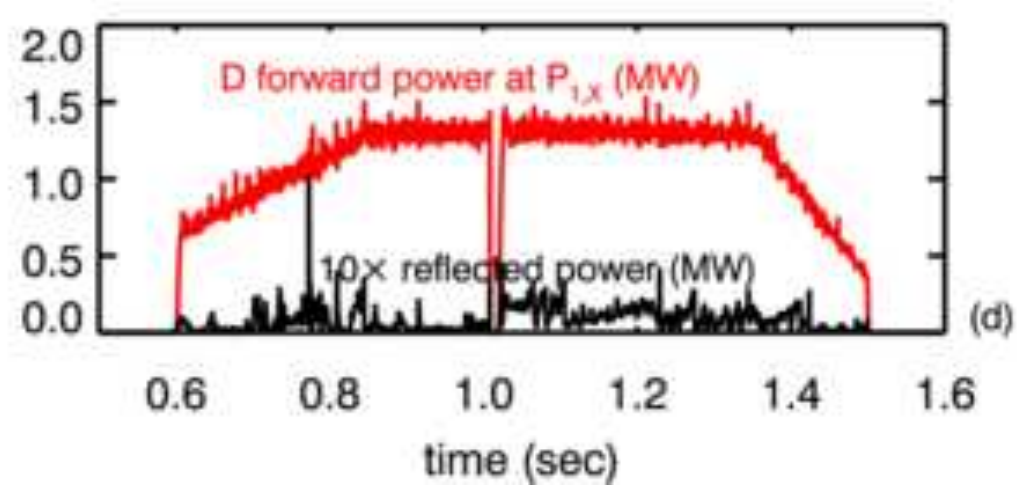

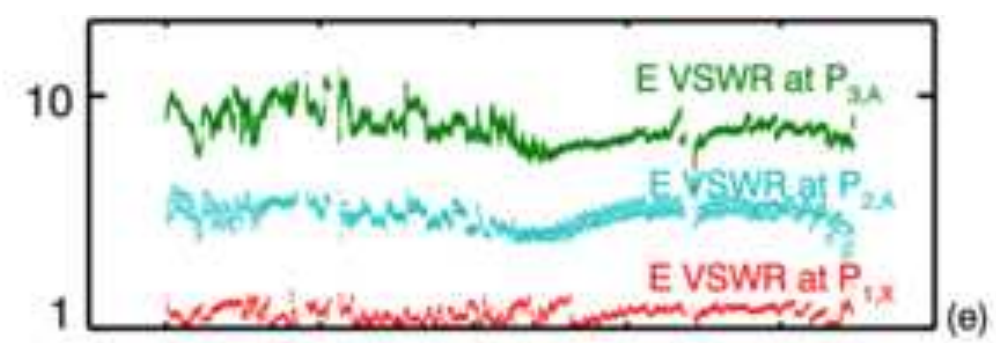
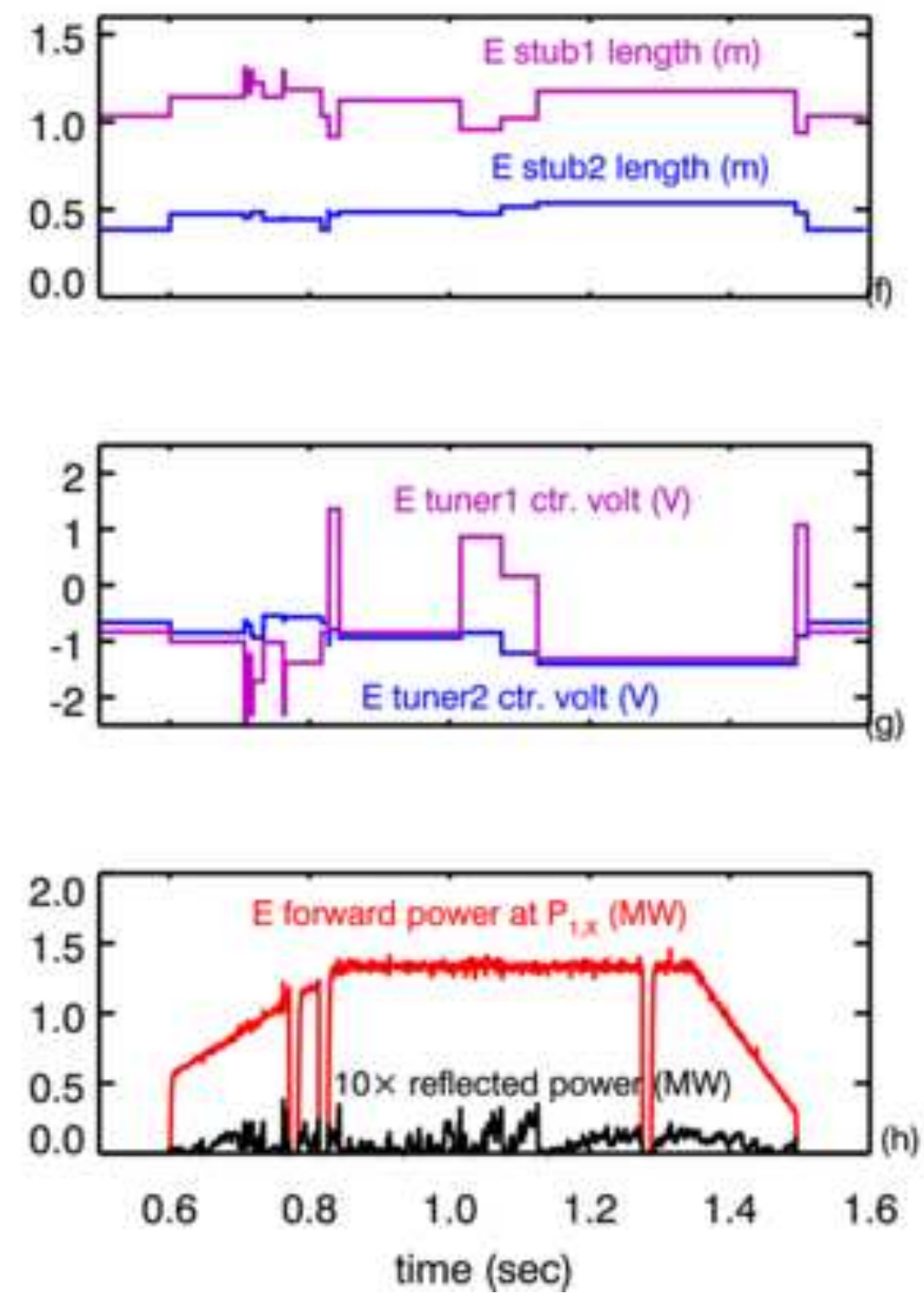

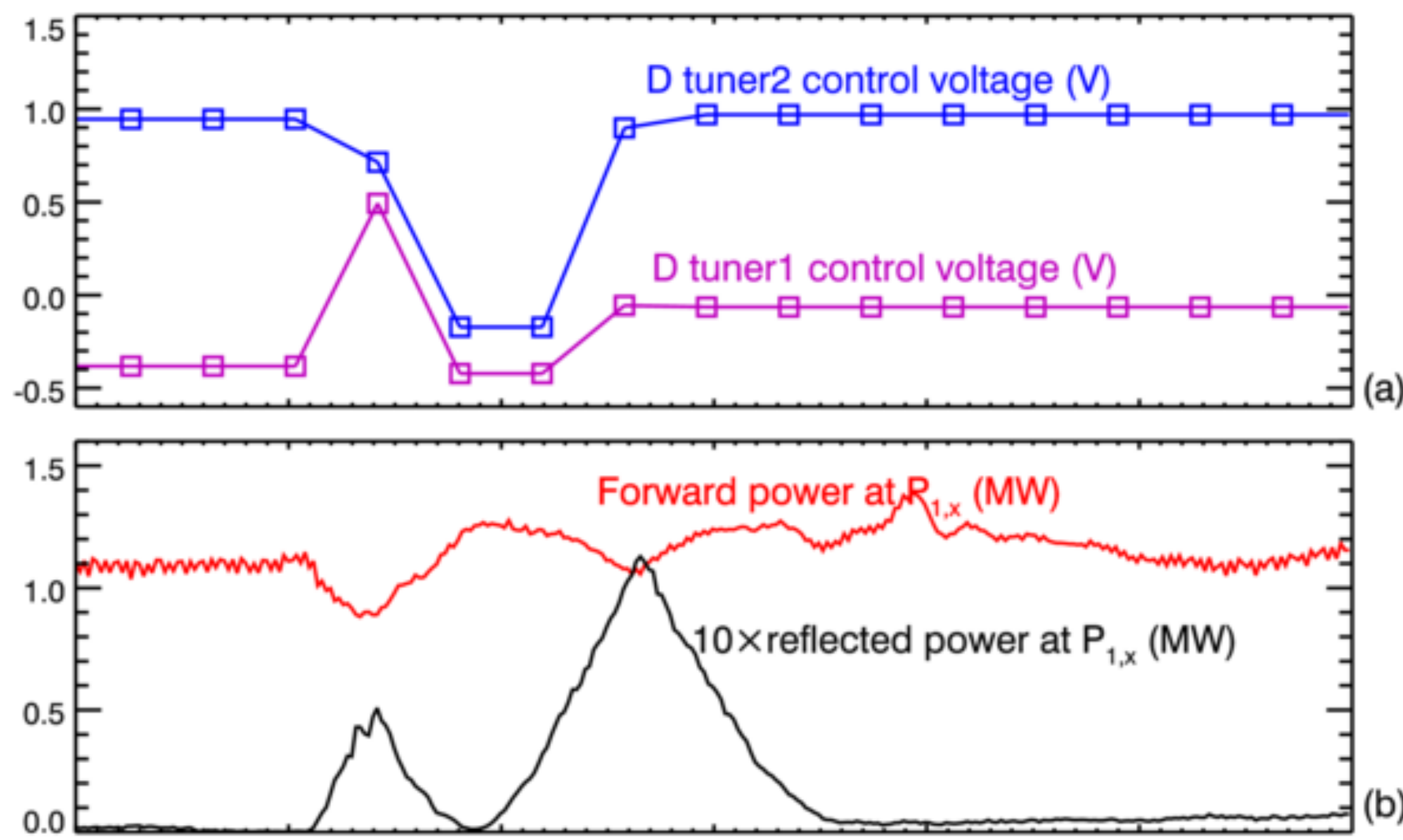

(b)

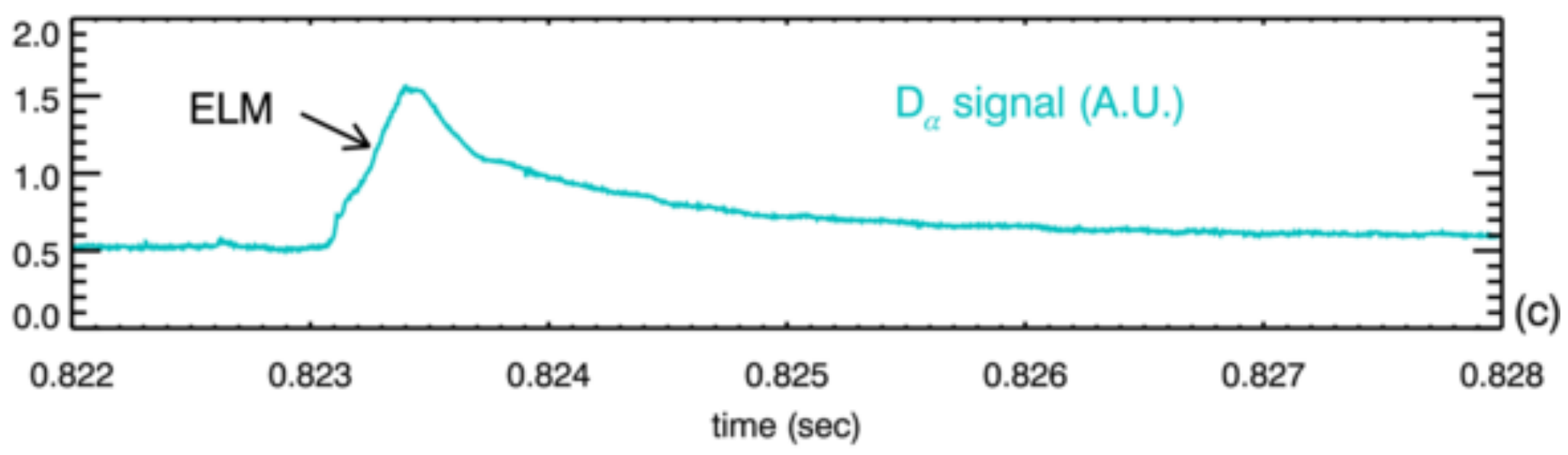



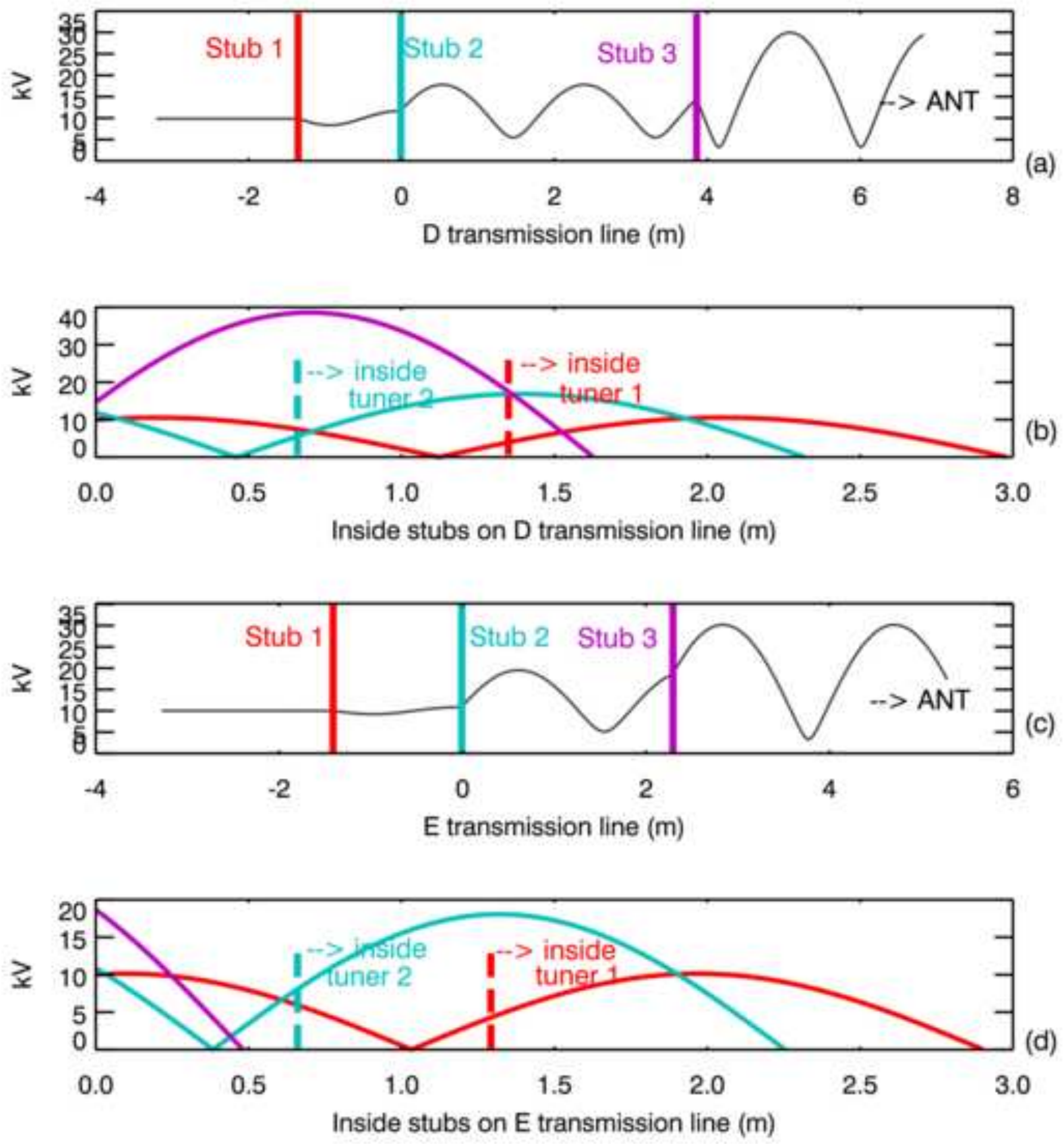
Smith Chart in $\mathrm{Y}=\mathrm{G}+\mathrm{jB}$

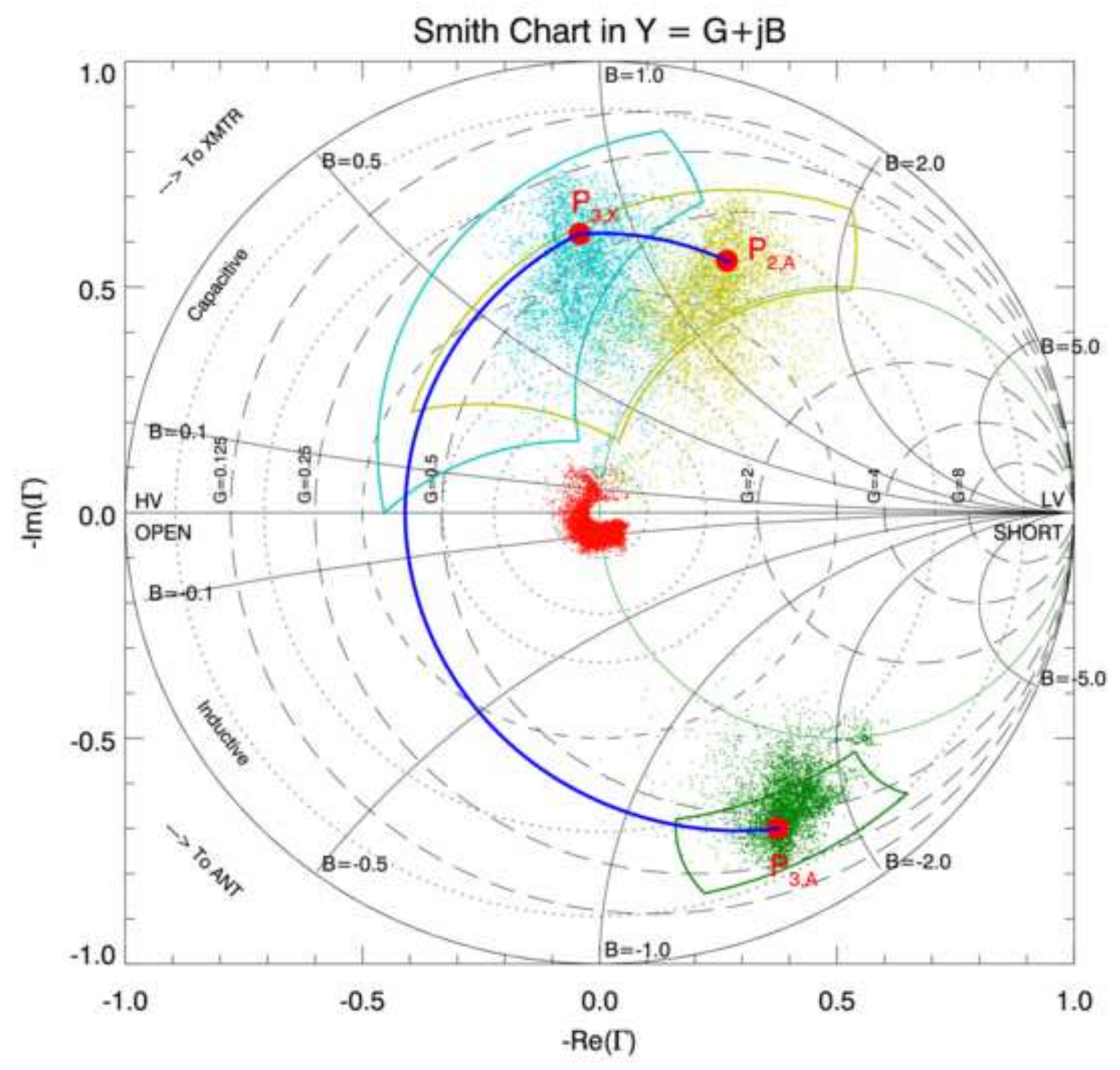

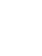




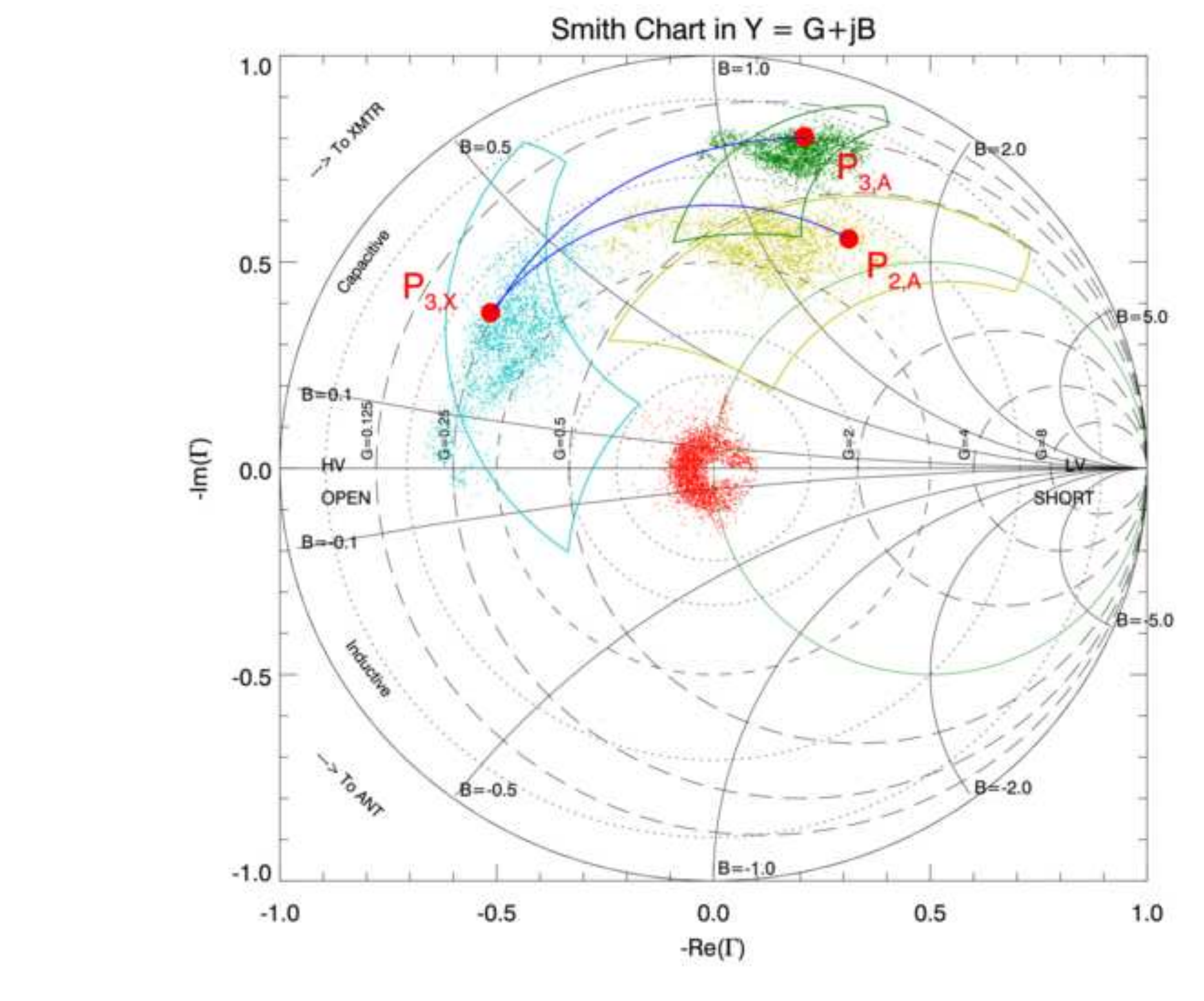

Smith Chart in $\mathrm{Y}=\mathrm{G}+\mathrm{jB}$

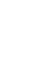

(n)




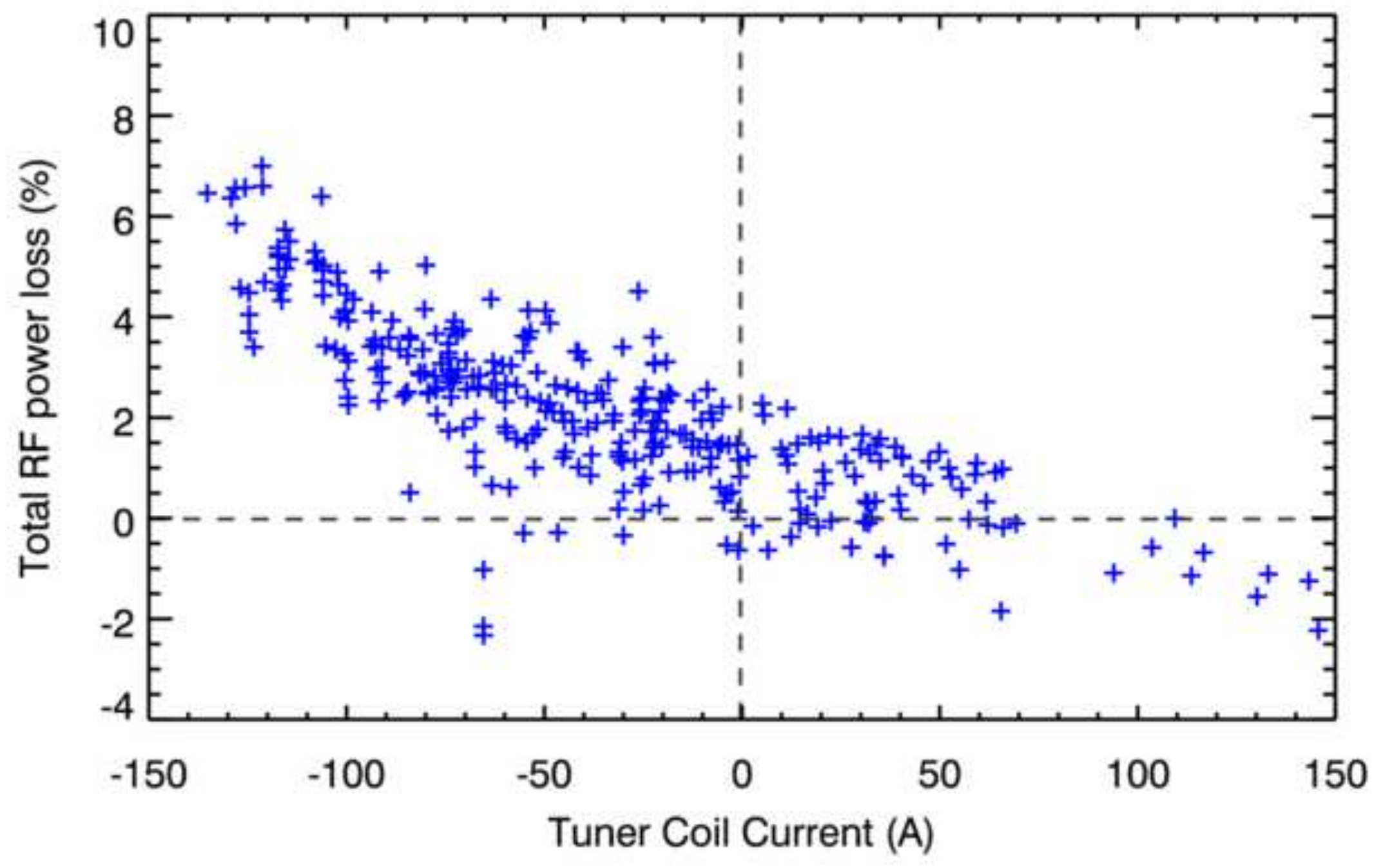




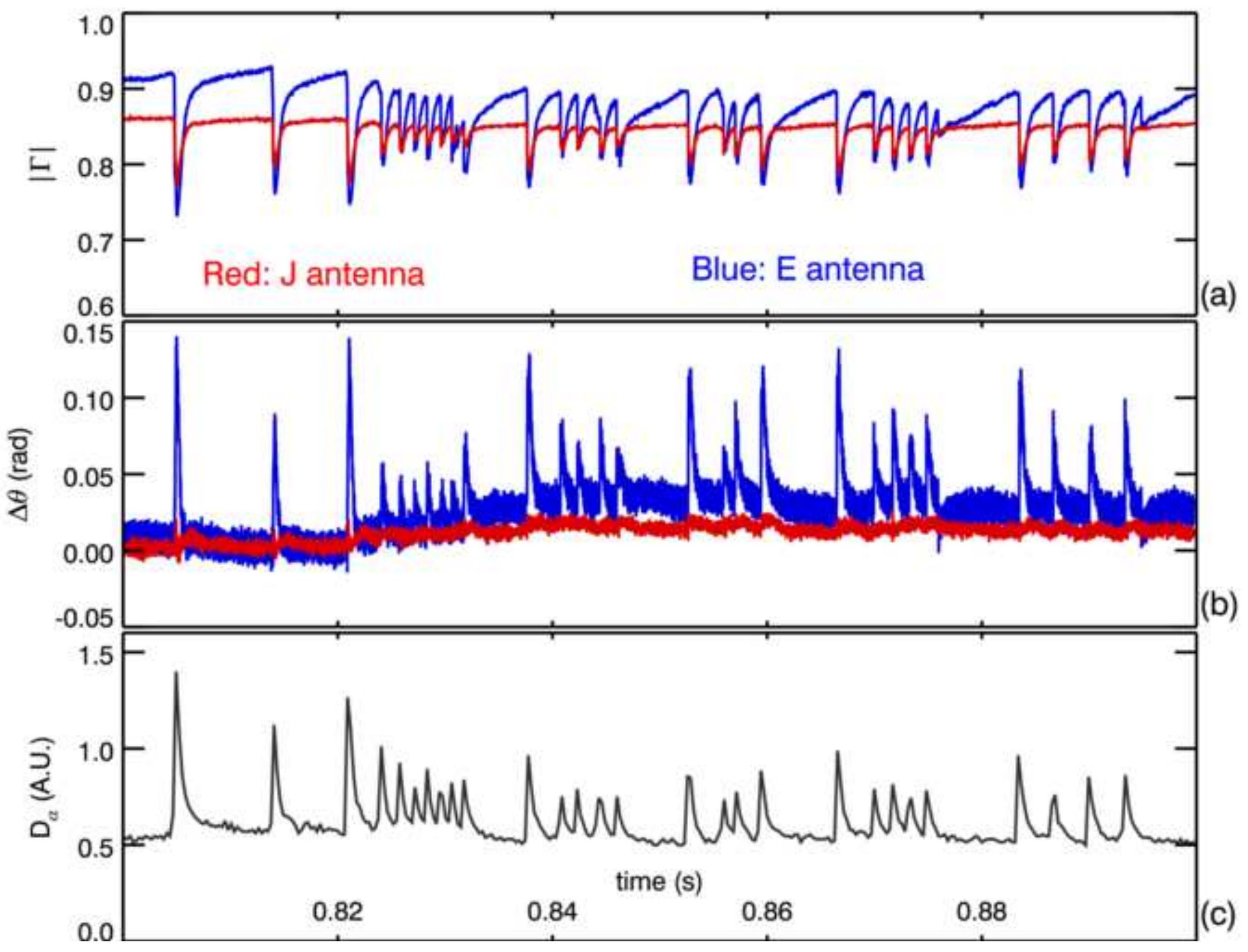




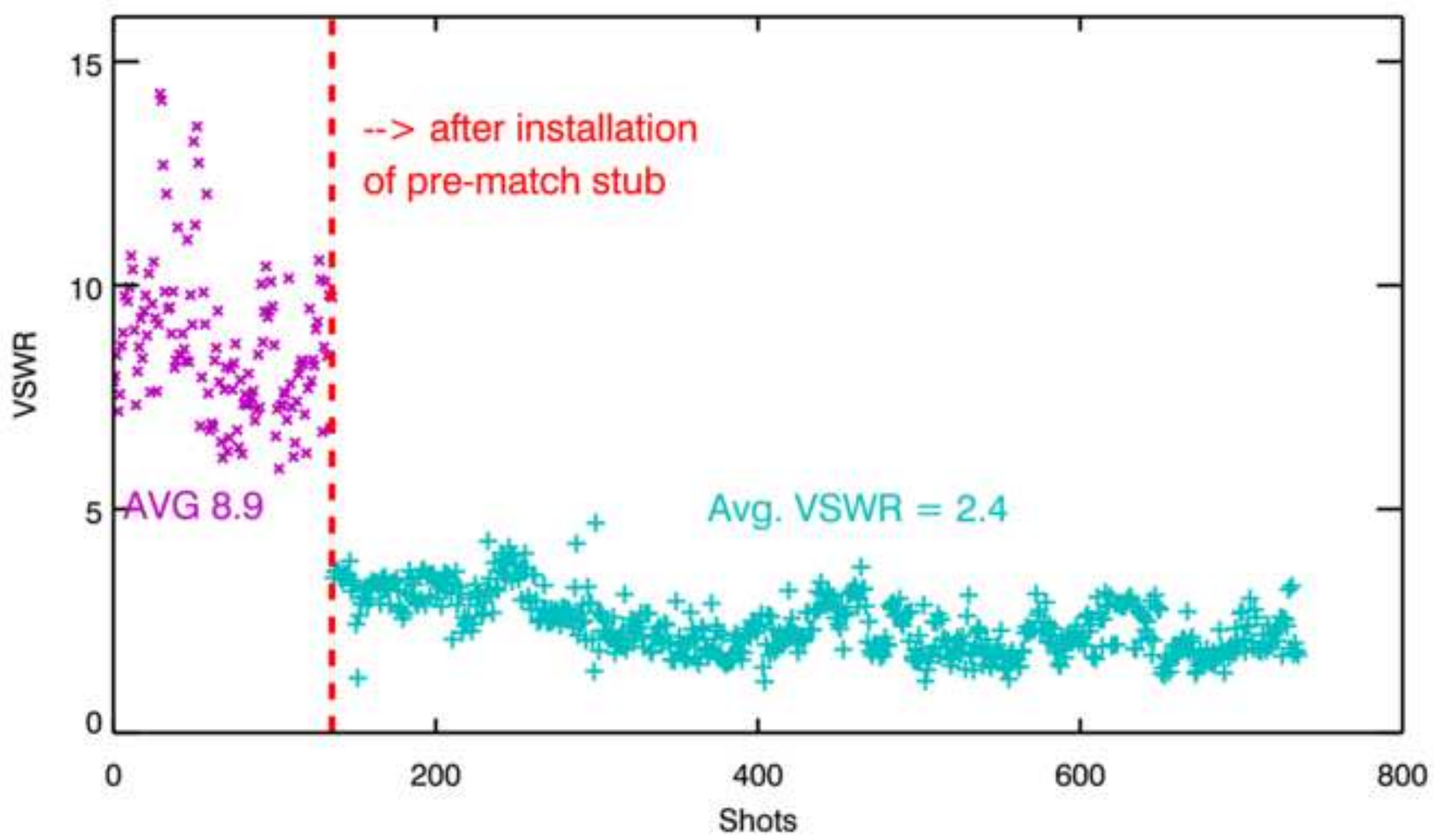




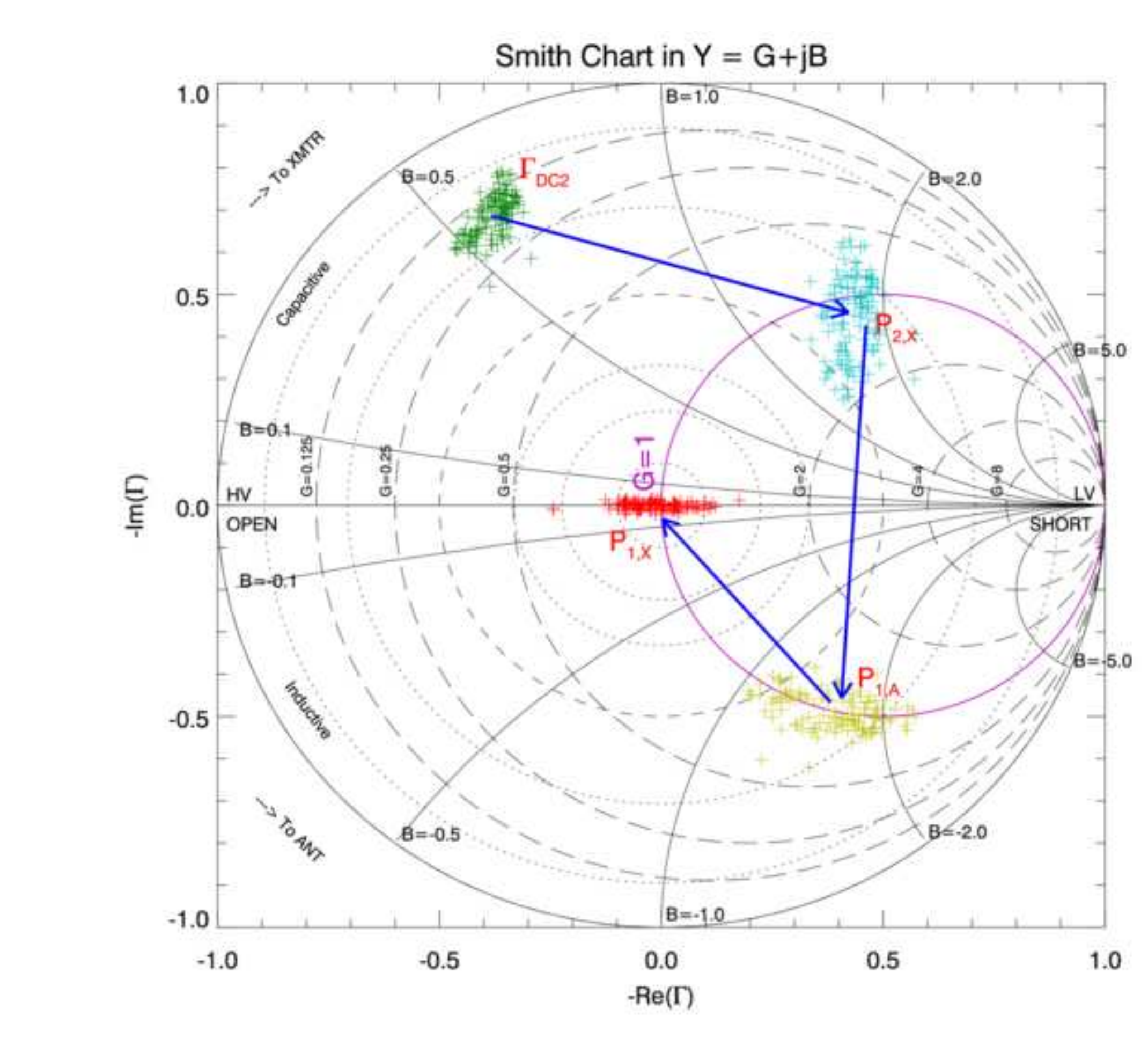

Smith Chart in $Y=G+j B$

Figure 13

.

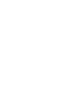

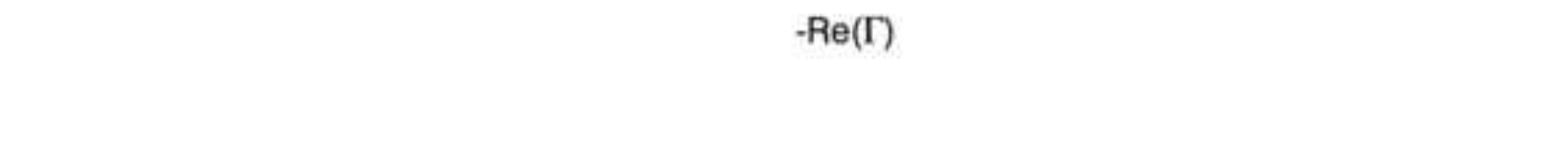

